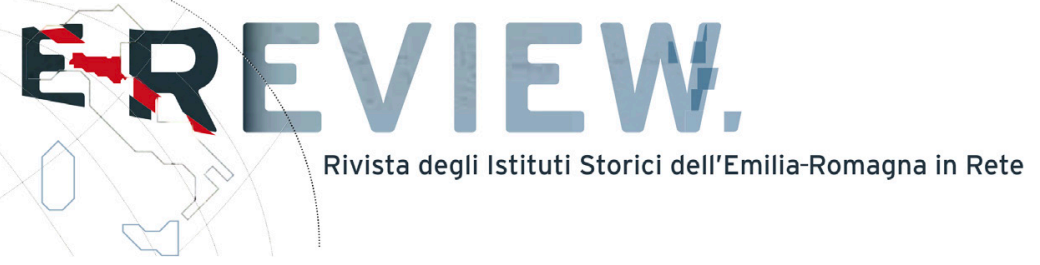

$\frac{\text { 8-9, 2021-2022 }}{\text { \#dossier }}$ 
E-REVIEW

Rivista degli Istituti Storici dell'Emilia-Romagna in Rete

Elena Pirazzoli

Sul piedistallo della storia. Statue innalzate, contestate, difese e demolite dalla Rivoluzione francese a oggi

(C) Elena Pirazzoli

Creative Commons BY-NC-ND 4.0

International License 2004-2021

Volume: 2021-2022

Issue: 8-9

Section: \#dossier - sul piedistallo della storia

Pages: $1-27$

DOI: $10.52056 / 9788833138756 / 07$

ISSN: 2282-4979

Publisher: Viella

Double blind peer review: No

Document type: Article

Research Areas: History

Published: December 2021

Corresponding Address: Elena Pirazzoli, Universität zu Köln, Martin-Buber-Institut für Judaistik, Albertus-Magnus-Platz, 50923, Köln, Germany 


\title{
Sul piedistallo della storia Statue innalzate, contestate, difese e demolite dalla Rivoluzione francese a oggi
}

\author{
ELENa Pirazzoli \\ Universität zu Köln - elena.pirazzoli@gmail.com
}

\begin{abstract}
Negli ultimi anni, dal Sudafrica agli Stati Uniti, dal Belgio all'Inghilterra, fino a dilagare per tutta l'Europa, sono sorti diversi movimenti per la rimozione di statue e monumenti, percepiti come simboli di un passato da cui prendere le distanze. Per comprendere questa ondata di contestazione è necessario allargare la visuale, ritrovando analogie e differenze con altre fasi iconoclaste accadute nella storia. Infatti, se l'atto dell'abbattimento è analogo, le intenzioni sottese possono essere molto diverse: sono contesto e prospettiva a definire se si tratti di vandalismo, di una dimostrazione di potere o di un processo di liberazione profondamente democratico. Questo dossier nasce da queste riflessioni, ancorate al dibattito del presente, cercando di individuare quali elementi e declinazioni peculiari si possano ritrovare anche nel territorio dell'Emilia-Romagna.
\end{abstract}

Parole Chiave: Statue, monumenti, POLITICA, POTERE, RIVOLTA

On the Pedestal of History. Statues Built, Criticized, Defended and Demolished since the French Revolution to the Present Day

In recent years, from South Africa to the United States, from Belgium to England, and spreading throughout Europe, various movements arose to remove statues and monuments, perceived as symbols of a past to be left behind. In order to understand these protests, it is necessary to broaden the view, finding analogies and differences with other iconoclastic phases that have occurred in history. While the act of demolishing is similar, intentions may be very different: context and perspective define whether it is vandalism, a demonstration of power or a deeply democratic process of liberation. This dossier is the result of these reflections, anchored in the present debate, trying to identify which elements and peculiar declinations can also be found in the territory of the Emilia-Romagna region.

KEYWORDS: STATUES, MONUMENTS, POLITICS, POWER, REBELLION

\section{Il ginocchio sul collo: gesti e simboli del potere razzista}

C’è qualcosa di mitologico nell'immagine del poliziotto con il ginocchio piantato sul collo della vittima a Minneapolis: San Giorgio che calpesta il drago sconfitto, la divinità purissima che schiaccia il serpente, perfino il cacciatore bianco sull'elefante o il rinoceronte ucciso in safari... Sono figure della vit- 
toria della virtù sulla bestia, dello spirito sulla natura, della civiltà sul mondo selvaggio... E del bianco sul nero. Così deve essersi sentito il poliziotto Derek Chauvin, domatore sul corpo prostrato di George Floyd in mezzo alla strada, davanti agli occhi di tutti.

Alessandro Portelli, Il ginocchio sul collo, 2020

Il 25 maggio 2020 George Floyd viene assassinato durante l'arresto dal poliziotto Derek Chauvin: un abuso di potere, un atto di violenza, di un bianco su un nero, che si va a inserire in una storia secolare di schiavismo, segregazione, linciaggi. A partire da Minneapolis, nel corso dell'estate 2020 si è diffusa un'ondata di proteste in tutti gli Stati Uniti: dapprima pacifiche, sono divenute sempre più dure, con scontri con la polizia e la Guardia nazionale, nonché con i suprematisti bianchi. A coordinare le proteste nelle principali città degli Stati Uniti è il movimento Black lives matter (Blm), sorto tra il 2013 e il 2014, in occasione delle uccisioni da parte della polizia di Michael Brown a Ferguson, in Missouri (colpito da diversi proiettili), e di Eric Garner a New York (anche lui immobilizzato e soffocato, morto ripetendo «I can’t breathe» - «Non riesco a respirare», parole riprese da Floyd e da altre vittime e che sono divenute uno slogan del movimento), fino al massacro nella chiesa episcopale metodista africana di Charleston ad opera di un terrorista suprematista nel 2015. Nato sotto forma di hashtag di indignazione e denuncia, Blm è divenuto poi un fenomeno politico fondamentale per le rivendicazioni della comunità nera americana dopo secoli di violenze. In particolare, è in conseguenza del massacro di Charleston che, non solo la comunità afroamericana, ma in generale una larga parte della società statunitense, ha cominciato a mettere in discussione i simboli confederati sparsi per il paese: segni razzisti che, soprattutto in alcune città degli Stati del Sud, hanno una centralità anacronistica, inaccettabile. Iniziano così a essere rimossi i monumenti - o meglio, quel particolare tipo di monumento figurativo che sono le statue - dedicati agli "eroi" sudisti: il presidente confederato Jefferson Davis, i generali Robert E. Lee, Thomas "Stonewall" Jackson, Nathan Bedford Forrest, ecc. Ma in questi territori, dove razzismo e segregazionismo hanno continuato a prosperare e a dare forma all'identità locale ben oltre la fine della Guerra civile, queste scelte sollevano le reazioni dell'ultra destra.

L'11 e il 12 agosto 2017 sono così i suprematisti bianchi a organizzare a Charlottesville, Virginia, nel quadro dell'Unite the right rally - raduno per unificare la galassia alt-right, nazionalista, razzista, neonazista statunitense -, una protesta contro la decisione del consiglio municipale di rimuovere la statua del generale confederato Robert E. Lee. Durante la contromanifestazione una donna è stata uccisa, e decine di persone sono state ferite, dall'irruzione di un'auto lanciata sui manifestanti da un giovane esponente della destra estrema.

Dopo questo evento, fino ad arrivare all'estate 2020, le proteste del movimento Blm e di altri gruppi antirazzisti sono divenute ancora più forti: principali obiettivi simbolici sono state proprio le statue confederate. Imbrattate, disarcionate, spodestate, le icone degli ex generali e politici sudisti sono divenute oggetto di quella 
Sul piedistallo della storia. Statue innalzate, contestate, difese e demolite

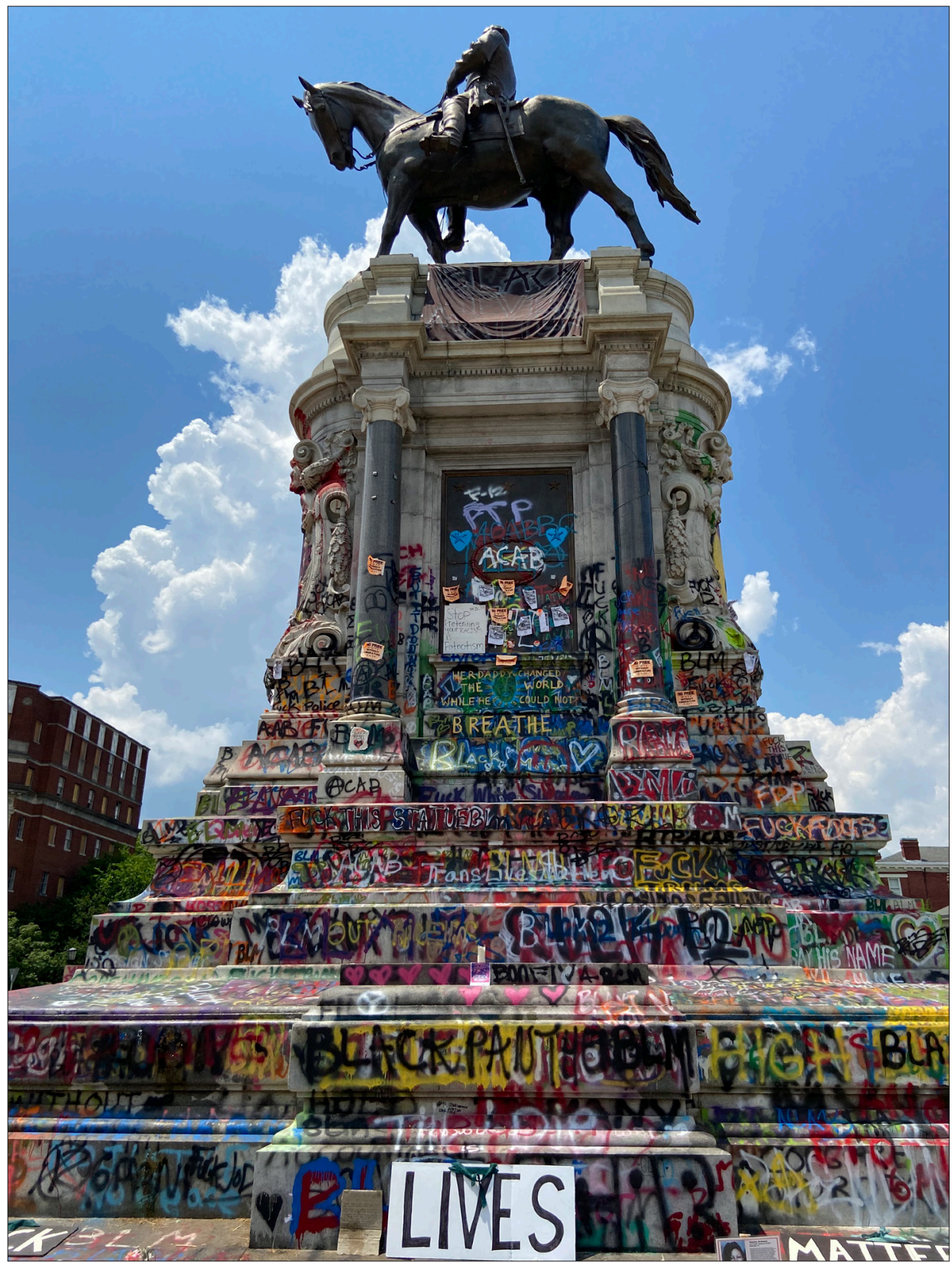

Fig. 1. Il piedistallo della statua di Robert E. Lee a Richmond (Virginia) ricoperto di scritte dopo le manifestazioni di Black lives matter, 1 luglio 2020 [foto Mk17b, CC-BY-SA 4.0]. 


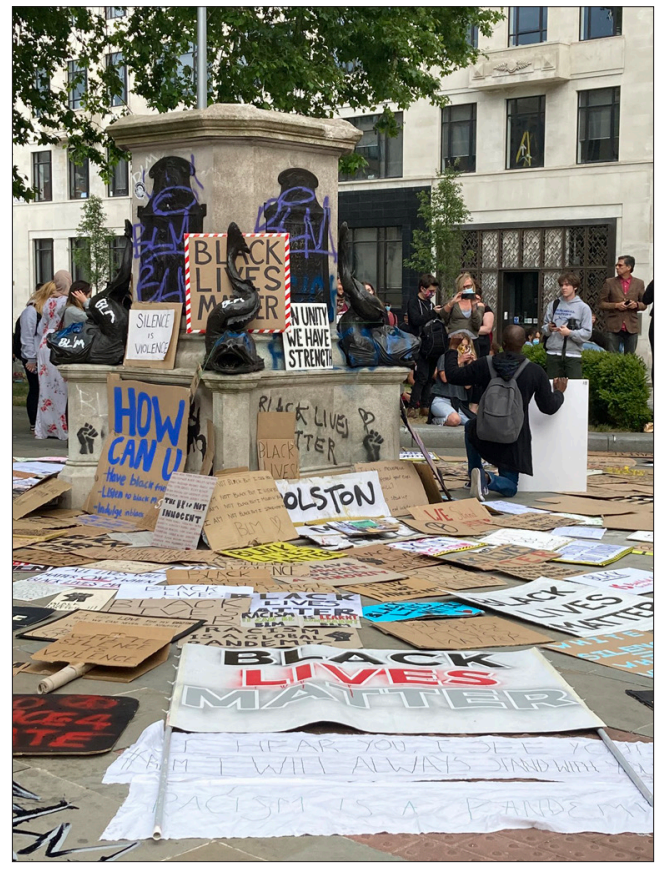

Fig. 2. Il piedistallo vuoto della statua di Edward Colston a Bristol, il giorno dopo l'abbattimento da parte dei manifestanti, 7 giugno 2020 [foto Caitlin Hobbes - CC-BYSA 3.0].

che è stata definita una "furia iconoclasta", una "guerra delle statue", denunciata come violenta dai media e da diversi esponenti del mondo politico. Perdendo tuttavia di vista come la violenza sui corpi abbia continuato a essere ben maggiore, ed evidentemente più grave, rispetto a quella sui simboli di marmo e bronzo. Dopo l'assassinio di George Floyd, infatti, altre centinaia di persone sono state uccise dalla polizia negli Stati Uniti: il database curato dal «Washington Post» riporta come lo scorso anno ci siano state 943 vittime in sparatorie, una su dieci disarmata, con un'incidenza di neri molto maggiore rispetto agli altri gruppi'

Il ginocchio sul collo, il peso sul dorso fino a spezzare il respiro e la vita: è questa l'icona dell'abuso violento del potere su chi ne è sprovvisto, questa posizione tenuta per interminabili minuti per immobilizzare il corpo del fermato, reo solo per presunzione. Ma colpevole per natura in un quadro di scontro razziale. Un'immagine della violenza, ripresa e diffusa sul web fino a diventare un'icona. O forse, una Pathosformel, una formula di pathos che, nella sua sistematica riemersione e riproposizione in latitudini diverse, mostra i corpi nell'atto dell'abuso di potere, il gesto di sopruso del dominante e l'agonia del dominato.

All'inizio dell'estate 2020, nel giro di poche settimane, l'ondata di proteste attraversa l'Atlantico e arriva in Europa, trasformando il suo carattere in un attacco contro i segni del colonialismo, ancora ben presenti nelle città europee,

\footnotetext{
Il database traccia il fenomeno dal 2015, dopo l'omicidio di Michael Brown a Ferguson, mostrando come il dato di circa 1.000 vittime l'anno sia stabile (https://www.washingtonpost.com/ graphics/investigations/police-shootings-database).
} 
Fig. 3. La statua di Indro Montanelli a Milano dopo le proteste dell'8 marzo 2019 [foto Bruna Orlandi].

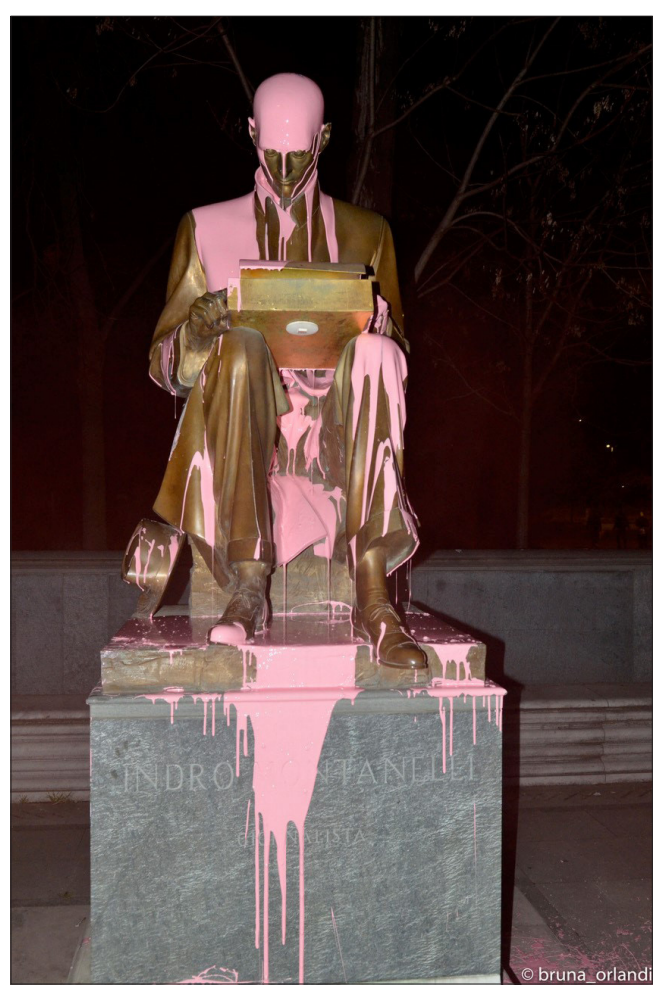

e tuttavia diversificati a seconda delle vicende storiche dei differenti paesi. In Inghilterra, Francia e Belgio, ad esempio, le manifestazioni nelle strade e le discussioni sui social media prendono di mira le statue dei mercanti di schiavi (come Edward Colston a Bristol e Robert Milligan a Londra), sovrani e politici che hanno governato l'impero coloniale o autori di legislazioni schiaviste (la regina Vittoria, Winston Churchill, Charles de Gaulle, Jean-Baptiste Colbert), ma soprattutto l'effigie di Leopoldo II del Belgio, ossessionato dal potere coloniale, da lui esercitato in forma di tirannia sanguinaria sul Congo, suo possedimento personale.

Negli Stati Uniti, intanto, il movimento Blm diventa sempre più ampio, inglobando istanze di altre comunità oltre a quella afro-americana, come i nativi, intrecciando così forme diverse di lotta al razzismo: contro lo schiavismo, il segregazionismo e il colonialismo. Dai confederati, l'obiettivo si sposta all'“origine” della questione coloniale, andando a colpire le effigi di Cristoforo Colombo, che nel frattempo viene celebrato alla stregua di un padre fondatore da Donald Trump nel discorso per il 4 luglio. Con buona pace di chi vorrebbe l'esploratore genovese incolpevole per tutto quello che è accaduto dopo il suo approdo, il peso di Colombo deriva da un mito fondativo creato negli Stati Uniti - dove egli non mise mai piede - alla fine del Settecento, dopo la Rivoluzione americana, nella necessità di darsi delle origini come uomini nuovi nel "nuovo mondo", in rottura con l'impero britannico. Nel peso simbolico dell'esploratore genovese, non è tanto il suo ruolo storico a contare, quanto questo investimento mitico: un uso - anzi, un 
abuso - funzionale a una legittimazione del potere bianco, libero, indipendente [Lorini 2020; Portelli 2020; ma anche Staiti 2018 per alcuni antecedenti]. In Italia, invece, l'eredità del colonialismo rimane tutt'ora qualcosa di poco percepito: anzi, possiamo parlare senza mezzi termini di una rimozione dalla storia nazionale, che comporta una totale mancanza di consapevolezza delle responsabilità italiane in Eritrea, Etiopia, Somalia e Libia. E anche laddove se ne abbia coscienza, il fenomeno è associato esclusivamente al fascismo, senza riconoscerne le radici nell'Italia liberale e le continuità di carattere economico nel secondo dopoguerra. Dato che il fascismo, crocianamente, dopo la sua fine andava messo tra parentesi, tanto più poteva essere messa da parte e dimenticata la fallimentare avventura coloniale italiana. Nell'estate 2020, le iniziative di protesta in Italia hanno così riguardato gli ingombranti monumenti del fascismo e i segni del colonialismo più noti e lampanti: il Foro italico e l'Eur a Roma, e la statua di Indro Montanelli nei giardini pubblici di via Palestro a Milano, a causa della famosa rivendicazione del giornalista - proferita anche in televisione con orgoglio e autocompiacimento - di avere usufruito della pratica del madamato, comprando in moglie una bambina eritrea quando era comandante di una compagnia di àscari nella Guerra d’Etiopia [Maida 2020; Scego 2020; Zucchetti 2020; Grechi, Lombardo 2021]². In questo quadro, però, stupisce come siano invece state minori, e con minimo spazio mediatico, le proteste contro il mausoleo a Rodolfo Graziani ad Affile (FR), realizzato solo nel 2012: viceré fascista dell'Etiopia, dopo aver subito un attentato scatenò la strage di Addis Abeba e quella di Debra Libanos, con migliaia di vittime ${ }^{3}$. La conoscenza di questi massacri - come delle violenze e della guerra chimica contro la popolazione locale - rimane ancora circoscritta a pochi in Italia: per lo più storici e attivisti.

Gli eventi statunitensi ed europei dell'estate 2020 hanno immediatamente e parallelamente innescato un intenso dibattito: sui social media come sulle testate giornalistiche o le riviste di approfondimento, da giugno ad agosto vi è stato un continuo susseguirsi di prese di posizione e discussioni accese, via via declinate in modo diverso a seconda del paese in cui avvenivano le proteste e gli "atti iconoclasti". Nell'autunno la discussione è approdata in ambito accademi$\mathrm{CO}^{4}$, mentre in quello mediatico si è estesa - ma in un certo senso potremmo dire

2 La statua di Montanelli era già stata oggetto di proteste l'anno precedente, durante la manifestazione dell'8 marzo organizzata dal movimento Non una di meno: in quell'occasione, per la prima volta, le era stata gettata sopra della vernice rosa. Un atto, più che iconoclasta, di “evidenziazione": l'effigie del giornalista non era stata distrutta, ma posta sotto un'altra luce per indurre a ragionare sulle sue scelte e posizioni, personali e allo stesso tempo contestuali, proprie di buona parte delle Camicie nere e dei soldati impiegati in Etiopia. Montanelli era solo uno dei tanti uomini complici di pratiche colonialiste e abusanti, e quella vernice rosa, posandosi sulla superficie incensante del monumento aggiungeva uno strato di significato e invitava a chiedersi come pensava e agiva quella generazione di italiani - ovvero i nostri nonni o bisnonni. .

3 Il monumento è stato imbrattato di vernice rossa l'anno successivo, in occasione del 25 aprile 2021.

4 Il dibattito mediatico dell'estate è stato ripreso a partire dall'autunno anche in ambito accademico: 
che è degenerata - verso il tema della cancel culture: uno sfuggente, complesso, manipolabile oggetto di dibattito pubblico, spesso presentato in modo confuso e alimentato dai sentito dire più che da approfondimenti dei diversi casi di presunta "cancellazione" ad opera del politically correct.

Questo dossier nasce in quel clima: la redazione di «E-Review» si è chiesta quali elementi, quali nodi, al centro della discussione e delle proteste potessero ritrovarsi, o avere dei corrispettivi, nel territorio - ovvero nella cultura, nella storia e nella memoria - della regione Emilia-Romagna. Da un confronto tra i vari rappresentanti degli Istituti storici della regione è emerso un quadro con caratteri solo in parte sovrapponibili a quanto stava avvenendo non solo in Europa, ma anche nel resto d'Italia. I saggi raccolti in questo dossier cercano di mettere a fuoco e analizzare le peculiarità di questo territorio attraverso alcuni casi studio e percorsi tematici.

Ma per dare un inquadramento al tema di attualità è necessario fare un passo indietro, per allargare la visuale ritrovando analogie e differenze con altre fasi iconoclaste. Perché se l'atto dell'abbattimento è analogo, le intenzioni sottese possono essere molto diverse: sono contesto e prospettiva a definire se si tratti di vandalismo, di una dimostrazione di potere o di un processo di liberazione profondamente democratico.

\section{Gocce d'olio su pietre roventi}

[...] la cosa più strana dei monumenti è che non si notano affatto. Nulla al mondo è più invisibile. Non c'è dubbio tuttavia che essi sono fatti per essere visti, anzi, per attirare l'attenzione; ma nello stesso tempo hanno qualcosa che li rende, per così dire, impermeabili, e l'attenzione vi scorre sopra come le gocce d'acqua su un indumento impregnato d'olio, senza arrestarvisi un istante.

Robert Musil, Monumenti, 1927

A inizio Novecento Robert Musil scrive un pungente testo sui monumenti, o meglio, sul destino di indifferenza che aveva avvolto i frutti della "monumentoma-

conferenze, seminari e progetti di ricerca hanno analizzato le molte questioni sollevate dalle contestazioni alle statue e ai monumenti, affrontandole dai diversi punti di vista disciplinari, dalla storia alla semiotica, dalla sociologia all'attivismo artistico. Per la stesura di questa introduzione si vedano in particolare il convegno Toppling Things. The Visuality, Space and Affect of Monument Removal, Universitat Pompeu Fabra di Barcelona, 21-21 gennaio 2021; il seminario dottorale Difficult Heritages: Monuments, Memories, Conflicts dell’Università di Genova, marzo-aprile 2021; la conferenza Come gira il vento. Monumenti, rappresentazioni, rappresentanza, con l'artista Luca Bertolo, Archivio d'Arte Contemporanea dell'Università di Genova, 14 maggio 2021; Statue abbattute e (ab) uso pubblico della memoria storica, seminario di Metodologia della ricerca storica dell’Università di Trento, gennaio-aprile 2021. Il tema era già stato esplorato in alcuni panel della seconda conferenza dell'Associazione italiana di public history (Pisa, 11-15 giugno 2018), in particolare AIPH23 Monumento in movimento: riqualificazione e risignificazione di opere monumentali in Italia; AIPH60 Lo spazio pubblico e i monumenti. 
nia” o "statuomania” del secolo precedente. Benché affollino le vie e le piazze, sebbene siano fatti per essere visti, giacciono tuttavia dimenticati, fallendo nella loro funzione immortalizzante. «Tutto quello che dura perde la forza di colpire» [Musil 2004, 63]. In realtà, questa perentoria affermazione è vera solo in parte: come abbiamo sottolineato, particolari urgenze del presente possono improvvisamente riportare lo sguardo su quei segni del passato che, visti sotto una luce diversa, prendono vita nel dibattito pubblico. Se la durevolezza può essere un paradossale ostacolo per l'incisività di un monumento ${ }^{5}$, è proprio la sostanza oleosa richiamata da Musil, a essere, per sua natura, infiammabile.

È accaduto più volte nella storia: sovrani, generali, conquistatori, uomini a cavallo o con l'arma in pugno, immortalati in ieratiche pose e immobili sui loro piedistalli da un tempo lungo (decenni, fino a secoli), sono stati improvvisamente disarcionati, spodestati, decapitati, nel migliore dei casi solo imbrattati. E la loro caduta è essa stessa parte della storia, segno di un cambiamento di equilibri di potere [Olusoga 2020].

L'elemento di novità portato dalla contemporaneità è l'ampiezza dell'ondata di proteste, capace di diffondersi da un continente all'altro.

A Cape Town nel marzo 2015 prende corpo il movimento Rhodes must fall per la rimozione della statua, collocata davanti all'Università locale (Uct), di Cecil Rhodes, imprenditore britannico, i cui spregiudicati affari furono la testa di ponte per l'avanzata colonialista inglese nei territori del Centro Africa e gli valsero l'incarico di primo ministro della Colonia del Capo. La proposta ha diviso l'opinione pubblica in Sudafrica, ma è stata accolta in aprile dall'Uct council, divenendo così il simbolo di un più ampio movimento di decolonizzazione nel paese, a partire dall'università (di cui Rhodes era stato un finanziatore). L'eco delle proteste, ma anche del loro più profondo significato culturale e politico, si è diffuso in altri paesi africani e nelle ex madrepatrie europee, come a Oxford - sede delle prestigiose e controverse Rhodes scholarships - poi in luoghi di colonizzazione "interna”, come la Groenlandia [Volquardsen 2020], infine negli Stati Uniti, andando a sovrapporsi alle istanze di Blm.

Come sottolinea Alessandro Portelli nel suo recente, lucido e prezioso saggio Il ginocchio sul collo. L'America, il razzismo, la violenza tra presente, storia e immaginari, siamo di fronte a un fenomeno storico: la presa di consapevolezza delle comunità nere, il loro desiderio di fermare la violenza e gli abusi a cui sono sottoposte da secoli, la richiesta di riconoscimento di un ruolo che necessariamente si scontra con quei simboli al centro delle piazze. Si tratta, riprendendo Ernesto De Martino, dell' «irruzione nella storia» di masse che fino a quel momento non hanno avuto voce nella narrazione storica, avendone tuttavia una: una storia all'ombra del gesto del dominatore [De Martino 1949, 411-435; Portelli 2020, 124].

\footnotetext{
5 La lunga durata offre il monumento all'indifferenza e all'oblio: in particolare in Germania l'arte di fine Novecento ha proposto, per fare fronte anche alla perdita di attenzione, casi di antimonumenti (Gegendenkmal) capaci di essere incisivi nel tempo breve e di relazionarsi con il pubblico, anche attraverso la propria scomparsa [Young 1992; 2000].
} 


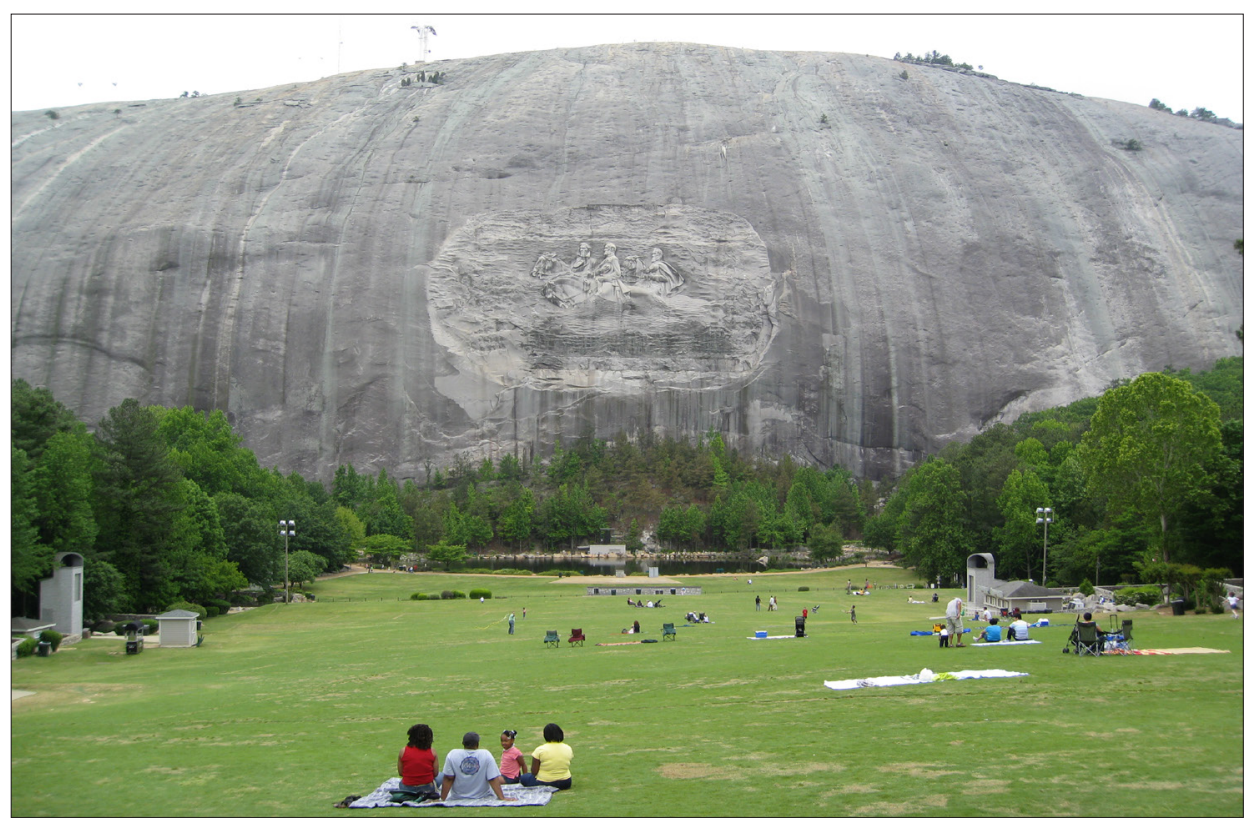

Fig. 4. Monumento confederato a Stone Mountain, Georgia, 2009 [foto Chris Younker - CC-BY-SA 2.0].

I poteri - in diverse culture, luoghi e tempi - hanno usato la retorica monumentale per dare corpo e immagine al proprio dominio: il monumento non dà semplicemente forma a un ricordo, quanto a un simbolo che deve legittimare il presente. Le figure rappresentate in quelle statue sono degli exempla: vengono dal passato, ma hanno un valore d'attualità. Sono moniti, modelli, per mantenere e dare forma all'ordine delle cose:

Le statue, gli obelischi, i nomi delle strade, delle piazze, degli edifici pubblici non sono tracce della storia, ma segni intenzionali con cui il potere presente afferma il proprio diritto di definire il significato del tempo storico e dello spazio pubblico. Non servono a ricordare che certe persone sono esistite ma a celebrarle e proporle come modelli normativi, ideali a cui ispirarsi [Portelli 2020, 109].

La retorica pubblica che disegna le città, o addirittura impronta una scala più ampia, paesaggistica (si pensi al Monte Rushmore, o al bassorilievo confederato a Stone Mountain, in Georgia) comunica, e impone, i propri contenuti anche a distanza di tempo. Ed è proprio il tempo ad avere un ruolo fondamentale nell'analisi del significato di questi monumenti. Nel caso statunitense, se si vanno a guardare le date in cui sono state collocate le statue attualmente oggetto di contestazione, si rimane stupiti dalla lontananza tra la loro erezione e l'evento che si vuole ricordare: sono fatte per il presente, per legittimare pratiche politiche radicandole in miti fondativi. Di conseguenza, è nel presente che vengono contestate, per i significati che affermano e impongono oggi. 


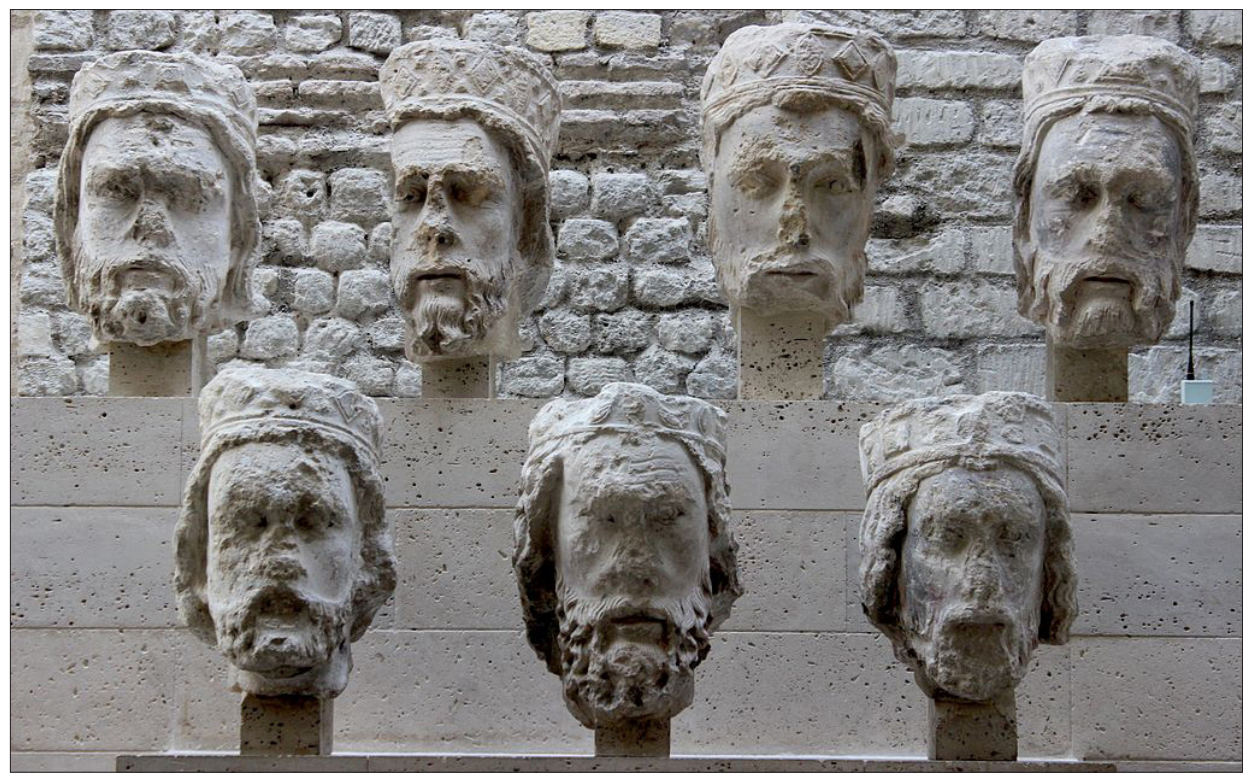

Fig. 5. Le teste dei re di Giudea della facciata di Notre Dame al Museo di Cluny [foto Miguel Hermoso Cuesta, 2013 - CC-BY-SA 4.0].

Un monumento esiste perché qualcuno l'ha eretto, e l'ha eretto in qualche momento con qualche intenzione: è un messaggio, un segno di quelle intenzioni. Quasi tutte le statue dei gerarchi sudisti non sono testimonianze del tempo in cui vissero e agirono, ma affermazioni delle politiche successive: la maggior parte sono state erette a cavallo del Novecento per sancire il consolidamento della segregazione razziale, negli anni Venti al tempo della massima espansione del Ku-Klux Klan, o ancora negli anni Cinquanta come reazione al movimento per i diritti civili [Portelli 2020, 119].

Quello che va a complicare la situazione nella nostra cultura occidentale, è il concetto di "patrimonio": il documento, il monumento, gli oggetti del passato con un rilievo storico, artistico e architettonico, sono divenuti a partire dal tardo Ottocento e in misura crescente fino a oggi, segni da preservare. Nel 1903 Alois Riegl in Der moderne Denkmalkultus (Il culto moderno dei monumenti), teorizza il tema del "valore" dei monumenti: valore artistico, valore storico, ma anche il valore per il presente. La prevalenza di un valore su un altro, e di conseguenza nelle scelte di conservazione, è un tema molto complesso, frutto di equilibrio tra preservazione del valore d'antichità e possibilità per i contemporanei di vivere il monumento in modo positivo e confortevole. Per determinare il valore di conservazione di un monumento, è quindi necessario analizzare queste funzioni commemorative storiche e discutere se esso contribuisca ancora alla narrazione collettiva in cui la comunità possa riconoscersi [Volquardsen 2020].

Nel caso dei monumenti confederati, per lo più il valore artistico è abbastanza scarso, mentre il valore storico è indubitabile - come documento - quanto unilaterale e impositivo - come monumento. In diverse occasioni, invece che 


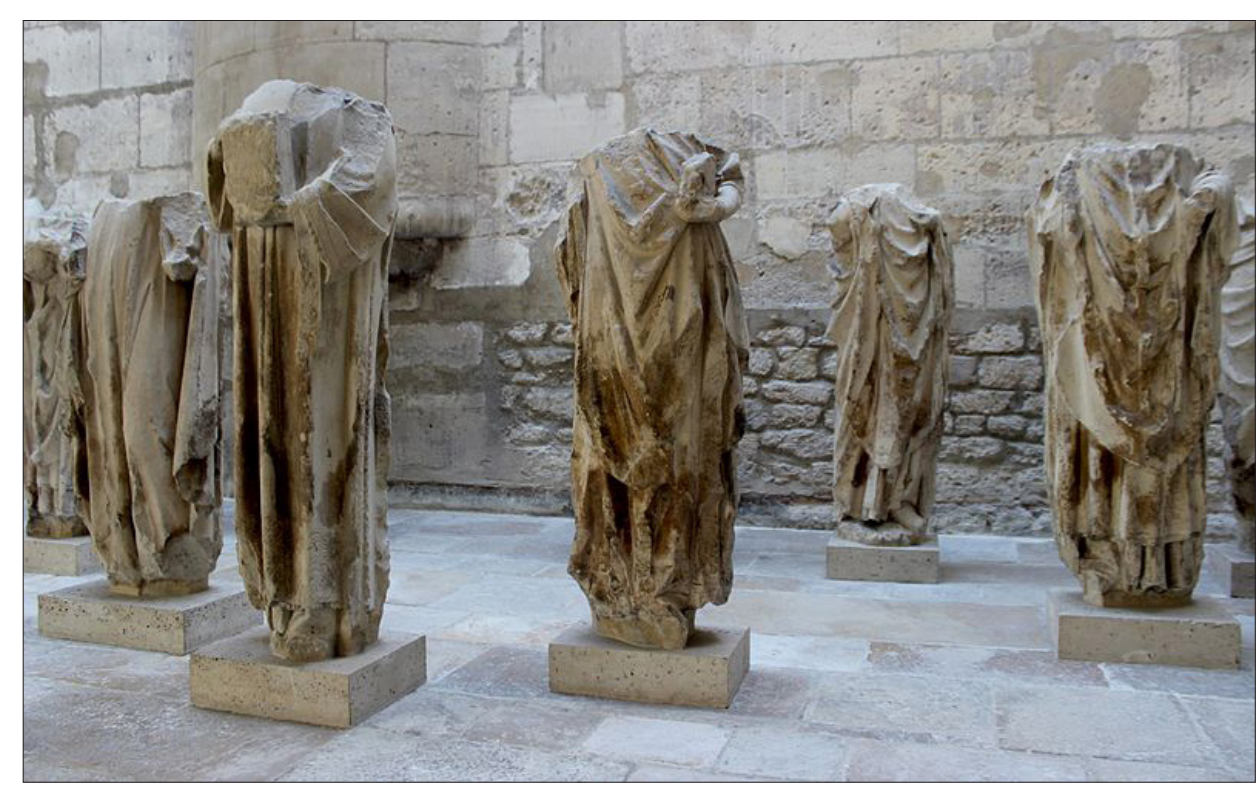

Fig. 6. Le statue decapitate dei re di Giudea della facciata di Notre Dame al Museo di Cluny [foto Miguel Hermoso Cuesta, 2013 - CC-BY-SA 4.0].

per "furore iconoclasta" le statue contestate sono state rimosse dopo un percorso istituzionale di riflessione sul loro effettivo valore per il presente, considerato divisivo e offensivo, se non eversivo. Un monito in cui non è più possibile riconoscersi, anzi. Se frutto di un percorso di questo tipo, i monumenti rimossi possono trovare nuove collocazioni che permettono di mostrare tutta la vicenda storica, sociale e politica, fino alla recente presa di consapevolezza sul loro significato. In particolare, questo può avvenire grazie a una ricollocazione in un luogo preposto a questa funzione conservativa e conoscitiva - come il museo - oppure grazie a interpretazioni - autoriali, artistiche o scientifiche - che permettono di costruirvi attorno una cornice risemantizzante.

Questo approccio è probabilmente debitore di una riflessione sviluppata a partire dagli anni Duemila in ambito teorico in particolare relativamente al patrimonio artistico e architettonico lasciato dai regimi fascista e nazista in Europa. Analizzando la complessità di relazione tra patrimonio e società, sono state coniate le due definizioni di dissonant e difficult heritage per quelle tracce del passato - per lo più recente - che provocano divisioni nel presente, per ragioni di mancanza di accordo, oppure per elaborazione mancata o ancora in divenire [Tunbridge, Ashworth, 1996; MacDonald 2009]. Tuttavia, negli Stati Uniti questo processo non appare così semplice: la sola persistenza negli spazi pubblici di monumenti nati per sostenere la supremazia bianca vale a ribadirne il messaggio, e anche lo spostamento in spazi museali in molti casi non ha funzionato come fattore di risemantizzazione. Il processo di "irruzione nella storia" delle comunità afroamericane e native è in atto, la migliore cornice risemantizzante al momento forse è proprio la vernice rossa sulle effigi dei simboli colonialisti: «When faced with a monument like this, viewers 

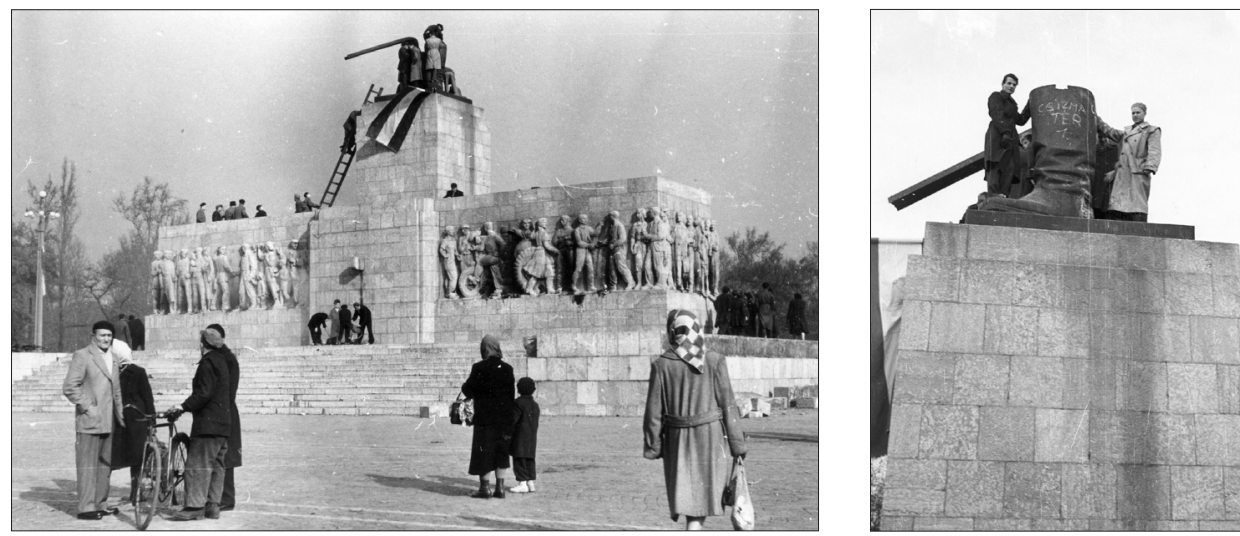

Figg. 7-8. Monumento a Stalin a Budapest dopo l'abbattimento della statua, 1956 [foto Fortepan / Pesti srác2 - CC-BY-SA 3.0 e Fortepan / Nagy Gyula - CC-BY-SA 3.0].

can't ignore the fact that not everyone agrees with the version of history that monument represents» [Thompson 2020; si veda anche 2021] .

\section{La caduta degli uomini di marmo}

Sembra che in Francia si possa fare una statua solo con le macerie di un'altra statua, e che se ne innalzi una solo per avere un pretesto per distruggerne un'altra.

Alphonse Karr, 1870 circa

Se abbattete i monumenti, risparmiate i piedistalli. Potranno sempre servire.

Stanisław Jerzy Lec, da Pensieri spettinati, 1957

L’abbattimento degli uomini di marmo si può considerare un topos, icona della rivoluzione e del sovvertimento del potere costituito che attraversa i secoli e le culture. Nell'antichità la damnatio memoriae era una pratica normata dal diritto romano, volta a cancellare figure di nemici vinti, in particolare i traditori, fino addirittura agli imperatori spodestati, affinché non potesse avere luogo nessuna riabilitazione presso la posterità, in cui non sarebbe rimasta alcuna traccia della loro esistenza. Se oggi si ragiona di diritto all'oblio, nell'antichità si metteva in atto la condanna all'oblio.

A questa pratica politica si è intrecciato, nel tempo, un livello più complesso, ovvero quello del sacro: dall'iconoclastia bizantina fino a quella protestante, per arrivare a quella islamica, il problema degli idoli e della raffigurazione del divino pone di fronte a una questione fondamentale, ovvero il potere intrinseco delle immagini.

6 «Di fronte a un monumento come questo, gli osservatori non possono ignorare il fatto che non tutti concordano con la versione della storia che questo monumento rappresenta» [traduzione di Elena Pirazzoli]. 
Un potere allo stesso tempo da reprimere - se idolatrico - e da usare, per la sua straordinaria immediatezza e capacità penetrativa [Freedberg 1989]. L'effigie del sacro, quindi, può essere oggetto di iconodulia e di iconoclastia: quest'ultima, a seconda dei casi, può essere una difesa estrema del sacro dalla sua idolatrizzazione, come, al contrario, un attacco al divino tramite la distruzione del suo simulacro.

Se si considera come la Rivoluzione francese sia stato il primo momento "moderno" di distruzione delle effigi del potere, non si può omettere di sottolineare come per secoli i sovrani francesi fossero stati considerati corpi sacri, re taumaturghi. Nella distruzione dei loro corpi fisici e simbolici c'è un'evidente dissacrazione, reificazione e umanizzazione di ciò che per secoli era stato considerato un potere intoccabile per la sua natura divina. Alla decapitazione di Luigi XVI seguì una profanazione: la distruzione delle tombe regali nella chiesa di Saint Denis. Ma una conseguenza fu anche la decapitazione delle statue degli antichi sovrani di Giudea dalla Galleria dei re della facciata di Notre Dame. È l'effige della “testa coronata”, il potere semantico di quell'immagine, a essere oggetto di distruzione. Queste statue, ritrovate fortuitamente nel 1977, sono oggi esposte al Museo di Cluny: i corpi e le teste sono ora documenti che testimoniano quella fase storica. Una rivoluzione produce piedistalli vuoti e macerie, che vanno a costituire gli elementi di un immaginario: quello della transizione verso un nuovo potere. Un immaginario in cui il potere è spodestato e sconfitto, le sue icone infrante e sostituite. I frammenti rimasti possono, in alcuni casi, diventare oggetti di storia: da monumenti divengono documenti, conservati nei musei per quello che possono raccontare anche nella loro distruzione.

Nella Francia nel 1793 questo processo accadde in parallelo alla fase iconoclasta: parte dei monumenti funerari reali di Saint Denis si salvò dalla completa distruzione e dissoluzione grazie all'intervento della Commissione delle belle arti, che acconsentì a farli confluire nella collezione del Musée des monuments français appena creato da Alexandre Lenoir proprio a partire dalle demolizioni ed espropriazioni rivoluzionarie. Il nuovo potere scelse cosa conservare, trasformando il monumento in documento, le effigi del potere sconfitto nelle tracce di quello che, d'allora in poi, divenne l'Ancien régime.

A quel momento di distruzione - di iconoclastia politica - ne seguì un altro, altrettanto eccessivo ed estensivo, di erezione di nuovi simboli, nuovi exempla. La "monumentomania" dell'Ottocento provvedette a sostituire le effigi del potere passato e sconfitto con quelle del nuovo e presente potere.

Il Novecento, quello europeo dei regimi, ma anche quello statunitense che seguì la Guerra civile, ha continuato a far proliferare questa retorica pubblica: propaganda monumentale figurativa e architettonica, quella che Hitler definiva «Worte aus Stein» (parole di pietra). Così, in Europa, alla caduta del fascismo e del nazismo, i loro simboli più evidenti - i fasci, le svastiche - furono scalpellati via o fatti saltare in aria. Se le effigi di Hitler furono oggetto di una vera e propria damnatio memoriae, in Italia, nel caso di Mussolini, la questione è più complessa [Ben-Ghiat 2017]. In alcuni territori si provvedette ad abbattere le sue statue subito dopo il 25 luglio 1943: così, ad esempio, a Bologna, dove il duce a cavallo allo 
stadio Littoriale fu disarcionato, la testa staccata e trascinata per le strade della città, poi nascosta e divenuta da allora oggetto di leggende. In altri casi, da Roma a Foggia, fino a Bolzano ${ }^{7}$, le effigi di Mussolini e del fascismo restano (o sono restate a lungo) negli affreschi e nei bassorilievi, per una sorta di inertia memoriae, come la definisce Mia Fuller ${ }^{8}$. Ed è solo sull'onda delle proteste dell'estate 2020 che alla fine di febbraio 2021 è stata rimossa nell'exclave di Melilla l'ultima effigie pubblica di Francisco Franco, applicando la legge spagnola del 2007 sulla memoria storica.

Un'intensa stagione di abbattimenti si ebbe in occasione delle rivoluzioni dei paesi dell'Est Europa contro i regimi comunisti: nel 1956 a Budapest, come nel 1968 a Praga, i monumenti a Stalin furono l'obiettivo simbolico principale delle insurrezioni. Distrutti, smembrati, i frammenti sparsi per le strade e i piedistalli vuoti rimasti come monito. Benché le rivolte siano state sedate, l'ordine costituito ripristinato, il potere delle immagini di quelle distruzioni è travolgente. Restano nella memoria e nella storia, divenendo icone.

La caduta del muro di Berlino il 9 novembre 1989 aprì un nuovo periodo di rimozioni in Europa orientale, con un carattere tuttavia diverso. Si trattò spesso di operazioni istituzionali, pianificate dai nuovi governi. In alcuni casi, come a Berlino e in altre città della Germania Est, gli abitanti si sono espressi contro queste rimozioni in quanto vissute come un atto esterno, che andava a cancellare segni della storia locale che si preferiva mantenere ed elaborare, per ragionare sul futuro della Germania riunificata a partire dal passato di divisione. Questa fase ha prodotto modelli di riflessione e di intervento estremamente interessanti, tra risignificazioni e risemantizzazioni ${ }^{9}$. In diverse occasioni gli interpreti di questo momento storico sono stati artisti.

7 Solo tre esempi: a Roma, nel salone d'onore del Coni presso il Foro italico, è stato riscoperto nel 1997 l'affresco di Luigi Montanarini Apoteosi del fascismo, celato dal 1944; a Foggia, sulla facciata del Palazzo di città, è ancora visibile il bassorilievo di Ermenegildo Luppi raffigurante Mussolini. In entrambi i casi non sono presenti spiegazioni o risignificazioni. Nel caso di Bolzano, invece, nel 2018 è stato realizzato un importante intervento di risematizzazione del fregio di Hans Piffrader sull'ex Casa littoria di Bolzano, in cui campeggia l'effigie di Mussolini a cavallo. Il progetto, a firma degli artisti Arnold Holzknecht e Michele Bernardi, si inserisce in un quadro più ampio di elaborazione delle tracce fasciste nella città, iniziato nel 2014 con l'inaugurazione dentro al monumento alla Vittoria del percorso espositivo BZ '18-'45 un monumento una città due dittature [Di Michele 2020; Pintarelli 2018].

8 Dal 2018 Mia Fuller sta portando avanti un progetto di ricerca dedicato a Mussolini Threshing still: Inertia Memoriae, Italy, and Fascist Monuments (https://nationalhumanitiescenter.org/fellow/ mia-fuller-2018-2019/). Parte delle sue riflessioni sono state oggetto dell'intervento Difficult how? Italy's Own Perplexed Treatments of Fascist Traces nell'ambito del seminario dottorale Difficult Heritages: Monuments, Memories, Conflicts dell’Università di Genova, 11 marzo 2021.

9 Nel 2016 è stata inaugurata nel Proviantmagazin della Zitadelle di Spandau la mostra permanente Enthüllt. Berlin und seine Denkmäler (Svelata. Berlino e i suoi monumenti: https://www. zitadelle-berlin.de/museen/enthullt/). Nel deposito della fortezza sono stati raccolti monumenti che, in diversi periodi storici, hanno modellato il paesaggio della città, ma sono stati successivamente rimossi dopo cesure politiche, in certi casi anche dopo essere stati attaccati e danneggiati. Statue 


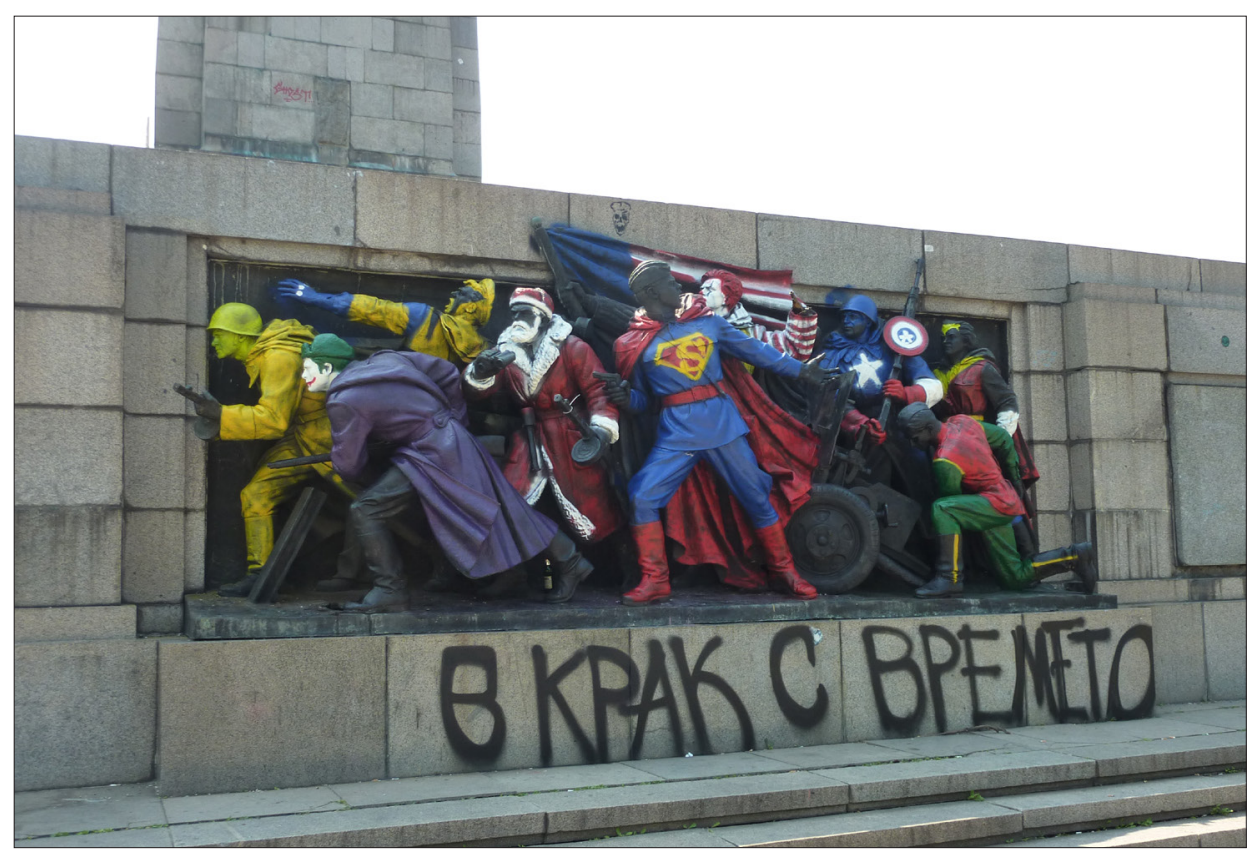

Fig. 9. Monumento dell'Armata rossa a Sofia, 18 giugno 2011. L'iscrizione significa: «al passo con i tempi» [foto Ignat Ignev - CC-BY-SA 3.0].

Nel 1991 Rudolf Herz inizia il progetto Lenins Lager, acquisendo dalla municipalità di Dresda il monumento a Lenin e trasformandolo in una propria scultura, che rimanda a «un deposito di un museo oppure un campo di macerie archeologiche», ovvero a «uno stato di aggregazione fra la distruzione e la ricostruzione museale» [Herz 1992, 5]. I frammenti di granito rosso riassemblati vengono successivamente portati dall'artista in giro per la Germania e l'Europa, caricati sul cassone di un camion aperto: con Lenin on Tour, Herz pone a passanti e visitatori la domanda «I show Lenin to my contemporaries. And I show Lenin the 21st century. Who will explain it to him?».

Qualche anno dopo, nel 2007, nella cornice dell'importante mostra di arte pubblica Skulptur Projekte - che si svolge ogni dieci anni a Münster - l'artista lituano Deimantas Narkevičius ha presentato un progetto irrealizzabile: Marx in Münster, che prevedeva di spostare la colossale testa di Marx da Chemnitz. Nel 1971 era infatti stato inaugurato a Karl-Marx-Stadt (come Chemnitz era stata denominata nel 1953) un colossale monumento: un basamento alto cinque metri e la testa alta sette. Nel 1990 la città riprese il suo nome originario, ma gli abitanti si rifiutarono di demolire o rimuovere la testa di Marx. Di fronte al diniego della municipalità, l'artista propose di farne una replica, ma anche questa idea venne rigettata: per la sindaca di

del potere prussiano, simboli del regime nazista, la grande testa del monumento a Lenin di Friedrichshain: radunati tutti nello stesso spazio, permettono di tracciare la storia politica e culturale di Berlino e della Germania. 


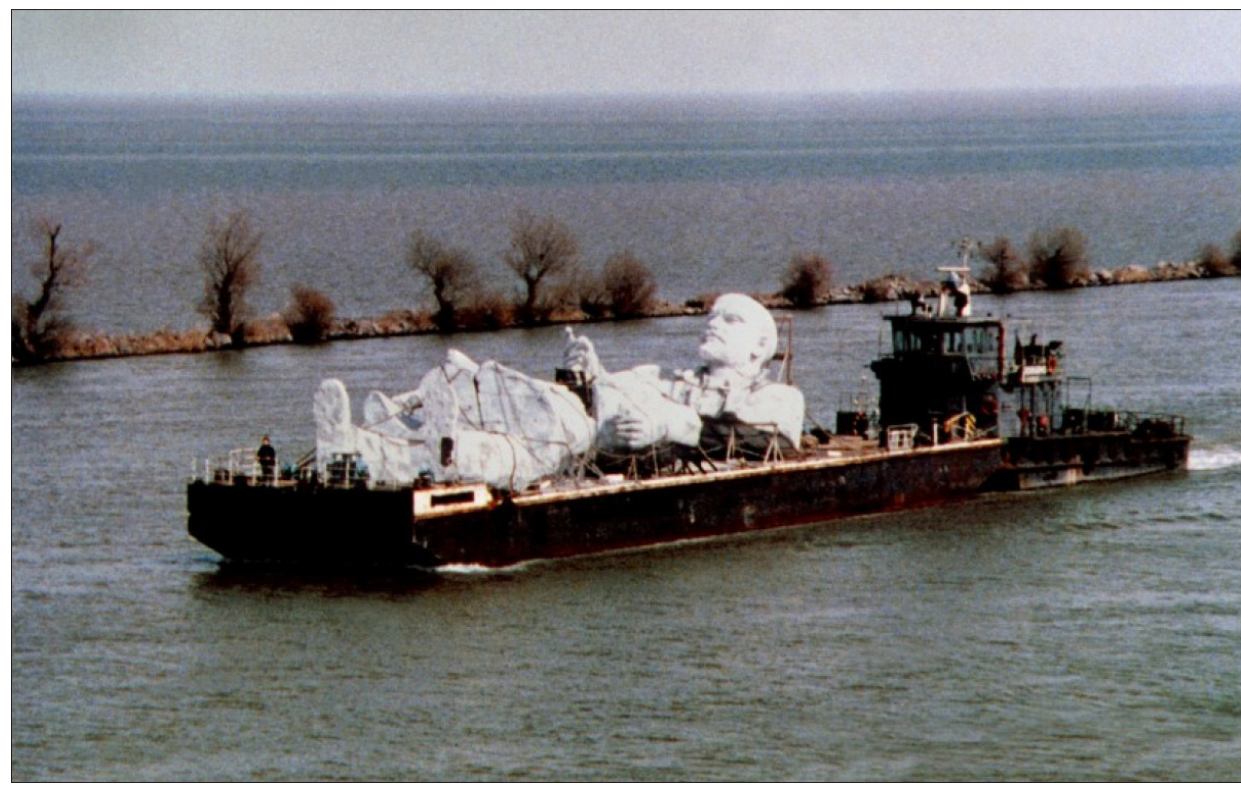

Fig. 10. Fotogramma da Theo Angelopoulos, Lo sguardo di Ulisse (Francia 1995).

Chemnitz il monumento è una copia unica, che va fruita nel suo contesto, storico e artistico. Narkevičius espose quindi in mostra The Head, un montaggio di filmati della televisione della Germania Est sulla costruzione e la ricezione di questo peculiare monumento ${ }^{10}$. L'anno successivo, gli studenti dell’Università di Arte di Linz, di Arti applicate di Schneeberg e di Scienze applicate di Merseburg crearono infine un percorso di visita attorno e all'interno della testa di Marx, intitolato Temporary Museum of Modern Marx ${ }^{11}$. In questo caso, il valore di quel monumento, il legame con la comunità locale e l'assenza di simboli esplicitamente riferiti a un significato traumatico, ha permesso di incorporare la statua in una narrazione storica che tenesse insieme il passato e la nuova prospettiva della Germania riunificata.

Nel caso del monumento all'Armata rossa di Sofia, in Bulgaria, invece, a partire dal 2011 si sono susseguiti diversi interventi di collettivi di attivisti: nel primo

10 Negli anni Sessanta lo scultore russo Lev Kerbel, a cui era stata commissionata la statua, affermò che «Karl Marx non ha bisogno di gambe o mani», sarebbe bastata la testa. Narkevičius, che nel 1990 era studente di scultura a Vilnius, ricorda come nel momento della ribellione della Lituania all'Urss in pochi giorni sparirono tutti i monumenti del recente passato sovietico, come per eliminare testimoni scomodi. «Tutti sembravano pensare che la rimozione di quegli oggetti avrebbe condotto a un immediato cambiamento della società», ma non fu così, sia perché il processo di trasformazione fu molto più lungo, sia perché qualcosa di quel passato è rimasto, insoluto, nelle radici del presente [Pirazzoli 2010, 178-179].

11 Molti di questi progetti artistici realizzati, o pensati, da artisti dei paesi dell'Est e dell'ex Urss dopo la cesura del 1989 sono stati oggetto della mostra Il piedistallo vuoto. Fantasmi dall'Est Europa, a cura di Marco Scotini, che si è tenuta a Bologna nel 2014. 


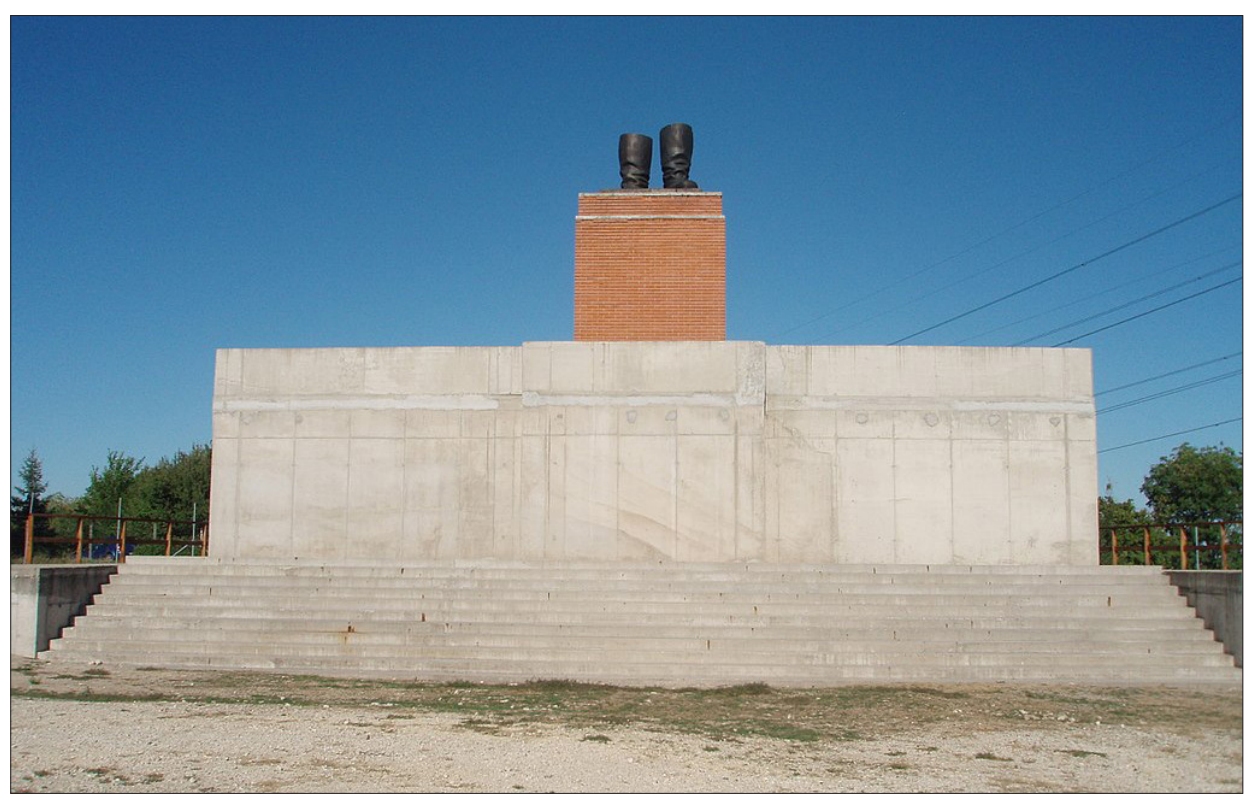

Fig. 11. Il basamento ricostruito con gli stivali di Stalin nello Szóbórpark di Budapest [foto Mister No CC-BY-SA 3.0].

caso, i soldati sovietici degli altorilievi sono stati dipinti trasformandoli in supereroi americani; nel 2012 sui loro volti è stata posta la maschera di Guy Fawkes, simbolo di diversi movimenti di protesta a partire da Anonymous; nel 2013 è stato dipinto di rosa per ricordare l'invasione di Praga; negli anni successivi è stata la volta dei colori della bandiera ucraina, polacca e bulgara, per evocare le politiche di violenta repressione da parte dell’Unione Sovietica [Panico 2017]. Ripulito dopo ogni "attacco" e nuovamente oggetto di intervento di risemantizzazione, il monumento, invece che essere abbattuto, si è rinnovato: agendo sulla sua superficie lo si è reso tutt'altro che "impermeabile alla vista".

L'icona più indelebile della stagione di rimozioni a Est è sicuramente la sequenza de Lo sguardo di Ulisse di Theo Angelopoulos (Grecia-Francia-Italia 1995) in cui un'enorme statua di Lenin, smembrata e ricomposta come un corpo nella camera ardente, passa su una chiatta su un fiume che attraversa i Balcani. Negli anni Novanta, nel pieno delle guerre jugoslave, la pellicola del regista greco intreccia la disgregazione del presente con l'eco delle violenze della Seconda guerra mondiale. Al passaggio della nave con quell'icona deposta e defunta, in viaggio verso la Germania per essere venduta a collezionisti, gli abitanti delle campagne corrono verso le rive, alcuni si fermano e si fanno il segno di croce ortodosso. Tra eventi storici e invenzione finzionale, è un ciclo che si chiude: quello iniziato con l'abbattimento della statua dello zar Alessandro III, ricostruito e messo in scena da Ėjzenštejn in Ottobre (Urss 1928). Il monumento distrutto diventa documento, allo stesso tempo relitto e reliquia: in alcuni casi le amministrazioni politiche di queste fasi di transizione hanno scelto di gestire il passaggio con consapevolezza. È il caso dello Szóbórpark di Buda- 
pest, il "parco delle statue”, rinominato poi Memento Park, costituito a partire dal 1991: i monumenti rimossi non vennero distrutti, ma "confinati” in un'area verde alle porte della città ${ }^{2}$. Busti di Lenin ed effigi di Béla Kun, eroi del lavoro, soldati dell'armata rossa, falci e martelli, stelle rosse, sono stati raccolti ed esposti in una modalità che dà la sensazione di trovarsi in un luogo a metà tra il parco a tema e il deposito di un museo. In particolare, la copia del piedistallo della statua di Stalin abbattuta nel 1956 si innalza nel giardino come potrebbe fare un'opera concettuale: un basamento di cemento e mattoni privato dei rivestimenti e delle decorazioni, con rimasti attaccati solo gli stivali di Stalin.

Le immagini di Stalin abbattuto e la folla festante nel 1956 a Budapest sono diventate un'icona, tanto da ispirare una, altrettanto famosa, "replica" [Mirzoeff 2004, 79-80]. Nell'aprile 2003 la colossale statua di Saddam Hussein a Baghdad venne abbattuta non dalla folla, quanto da uno sparuto gruppo di persone, ma in favore di telecamere e macchine fotografiche dei reporter embedded che seguivano la presa della città da parte delle truppe americane. L'operazione di propaganda voleva evocare sentimenti di trionfo sul nemico, di liberazione dal tiranno, utilizzando il "potere delle immagini”. Tale potere, infatti, non emana solo dalle statue erette, ma si ritrova anche, per opposto, nelle immagini che colgono l'istante della loro caduta ${ }^{13}$.

Lo storico dell'arte Dario Gamboni - studioso della distruzione delle immagini monumentali del potere a partire dalla Rivoluzione francese - sottolinea questo aspetto cruciale:

La coincidenza della caduta del Muro di Berlino con il duecentesimo anniversario della Rivoluzione francese rende il paragone inevitabile. Come la Bastiglia, il Muro è stato distrutto (quasi) interamente, sebbene in una maniera meno organizzata, ed è stato trasformato in reliquie e souvenir. Immagini della rimozione 0 distruzione di monumenti hanno prodotto la più drammatica ed efficiente visualizzazione delle trasformazioni politiche di quello di cui erano parte, e si è affermato che almeno in alcuni casi, il loro appeal sui mass media è stata una delle ragioni per cui esse [distruzione e rimozione] sono accadute [Gamboni 2002, 98].

Questo brano è pubblicato nel catalogo di un'importante mostra realizzata nel 2002 a Karlsruhe: Iconoclash. Beyond the Image-Wars in Science, Religion and Art era

12 Nel 2007 il Memento Park è stato ampliato con l'aggiunta di sale espositive in cui viene raccontata l'insurrezione del 1956 e le vicissitudini politiche fino al 1989-1990. Altri parchi analoghi sono stati realizzati in altri paesi dell'Europa orientale: per esempio il Grutas Park in Lituania e il Museo dell'arte socialista a Sofia, in Bulgaria.

13 Si pensi alla famosa stampa de «L'Illustration» che coglie l'abbattimento della colonna Vendôme a Parigi nel 1871, come se si trattasse di un'istantanea. Esistono diverse fotografie di quel momento ma, data l'impossibilità tecnologica di effettuare scatti che potessero cogliere l'istante, esso fu ricreato da un'incisione. Per una riflessione, si veda la conferenza Come gira il vento. Monumenti, rappresentazioni, rappresentanza, con l'artista Luca Bertolo, Archivio d'arte contemporanea dell’Università di Genova, 14 maggio 2021. 
articolata come una complessa riflessione scaturita, anche in quel caso, da eventi dell'attualità di portata epocale. ll nuovo secolo, e millennio, si era infatti aperto con l'abbattimento delle Twin Towers a New York: simbolo del potere occidentale, trasformato con l'attacco terroristico e la diffusione - in diretta mondiale del crollo delle torri in icona della caduta, o quanto meno della radicale messa in discussione, dell'equilibrio geopolitico vigente. La fase di transizione aperta in quel momento è ancora in atto: la nuova crisi in Afghanistan di agosto 2021 ne è una terribile dimostrazione. La ripresa del controllo del paese da parte dei Taliban richiama alla mente quale icona scelsero vent'anni fa per mostrare il loro potere: l'abbattimento dei Buddha di Bamiyan, icone sacre in contrasto con le linee più fondamentaliste dell'islamismo. Atti di iconoclastia che producono, anzi, ripresi e diffusi sugli schermi, “diventano” essi stessi le icone del nuovo potere.

\section{In Emilia-Romagna: tra eredità difficili, partecipazione pubblica, imperdonabili assenze}

Bologna ha il monumento ai caduti più straordinario che ci sia. Orribile ma perfetto. Dal punto di vista estetico, vale meno di zero, ma questo non cambia nulla. È un muro, [...] e il nome di ogni morto è illustrato dalla sua fotografia, la fotografia fornita dalla sua famiglia. Ci appaiono dunque com'erano agli occhi di chi li amava. [...] Mi sono venute le lacrime agli occhi davanti a un nome che era stato illustrato da una madre [...] con la fotografia di un biondino in braghini corti e colletto alla marinara. Aveva voluto custodirlo nel ricordo a quell'età. Mi sono avvicinato all'immagine, sia per mascherare la mia emozione, sia per imprimermi nella memoria i tratti di quel bambino. Era ancora più terribile di quanto pensassi. Si trattava della foto di un cresimando, pieno di meraviglia. [...] Questi fantasmi disposti lungo il marciapiede, in uno dei luoghi più frequentati della città e così com'erano nella loro umile vita, sono più commoventi di tutti i grandi ordini architettonici.

Jean Giono, Voyage en Italie, 1954

Quali elementi di questa complessa genealogia di erezioni e distruzioni, rimozioni ed elaborazioni si ritrovano nelle vicende dell'Emilia-Romagna? Sull'onda delle proteste negli Stati Uniti e nel resto d'Europa nell'estate 2020 anche in questa regione sono sorte diverse iniziative per mappare le tracce del colonialismo: per lo più rimosse dalla coscienza nazionale e quindi non viste neppure a livello locale, si ritrovano soprattutto nella toponomastica, nelle lapidi delle "inique sanzioni” [Wu Ming 2 2020] e nelle architetture degli anni Venti e Trenta ${ }^{14}$. In molti

\footnotetext{
14 In regione è stato dedicato all'analisi e a una riflessione pubblica sul dissonant heritage del Ventennio fascista il progetto Atrium di Forlì (https://www.atriumroute.eu/). A livello nazionale, si veda anche il progetto di ricerca dell'Istituto nazionale Ferruccio Parri Luoghi e memoria del
} 


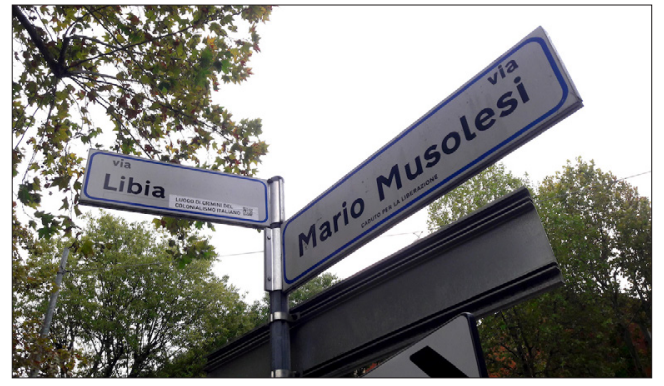

Fig. 12. Guerriglia odonomastica in Cirenaica a Bologna [foto Elena Pirazzoli, 2021].

casi, in Emilia-Romagna dopo il 1945 questi segni sono stati trasformati o ridenominati in favore della Resistenza. Un'operazione di risemantizzazione dovuta alla vittoria sul fascismo, ma che tuttavia ha prodotto anche l'occultamento delle tracce del colonialismo italiano, celebrato dal regime e di conseguenza epurato dalle memorie liberate. Come si può uscire da questo paradosso?

Nel contesto della riflessione statunitense attorno agli interventi di carattere più performativo di Blm si è parlato di art of removal: "arte della rimozione", che interviene creando nuovi segni sui piedistalli rimossi. Una strategia utilizzata anche da alcune esperienze di attivismo locale, volte a mostrare gli strati di storia depositati su quei segni. Uno dei casi più interessanti, attivo da alcuni anni a Bologna, è il collettivo Resistenze in Cirenaica, che raccoglie e racconta sia le storie di Resistenza cittadina a partire dal rione Cirenaica (così denominato in quanto costruito a partire dal 1912 e quindi per omaggiare la Guerra di Libia e i suoi "successi", le cui vie sono state quasi tutte rinominate nel 1948 e dedicate a partigiani), sia quelle delle Resistenze contro le occupazioni coloniali italiane ${ }^{15}$. Una delle pratiche messe in atto è la "guerriglia odonomastica", ovvero la rinominazione o specificazione delle vie cittadine.

Per molti aspetti, quello che accade in questa regione da metà XIX secolo non si discosta dalle scelte di retorica urbana del resto del paese, ridisegnata dopo il Risorgimento e l'Unità in linea con la monumentomania caratteristica di tutto l'Ottocento europeo. All'erezione di statue per i "padri della patria" - Vittorio Emanuele, Cavour, Mazzini e Garibaldi - e alle figure locali che si erano distinte nella lotta per l'indipendenza, seguirono poi le retoriche urbane dell'Italia liberale e, soprattutto, quelle del fascismo: dagli eroi della Grande guerra a quelli delle imprese coloniali, ai gerarchi nazionali e locali. Una nuova stagione di monumentomania i cui prodotti divennero oggetto di attacchi dopo la caduta del regime, in questo territorio come in tutta l'Italia centro-settentrionale, quella che ha vissuto i diciotto mesi della Repubblica sociale: le statue, i monumenti e

fascismo coordinato da Giulia Albanese (https://www.reteparri.it/ricerca/progetti-di-ricerca/luoghimemoria-del-fascismo/).

15 Si veda il sito Resistenze in Cirenaica (https://resistenzeincirenaica.com/) Tra le altre esperienze regionali si segnalano le attività degli Arbegnuoc urbani a Reggio Emilia. 
Fig. 13. Statua disarcionata di Mussolini a cavallo allo stadio Littoriale [foto Nino Comaschi, 1945 circa, Archivio Fotografico, Cineteca di Bologna].

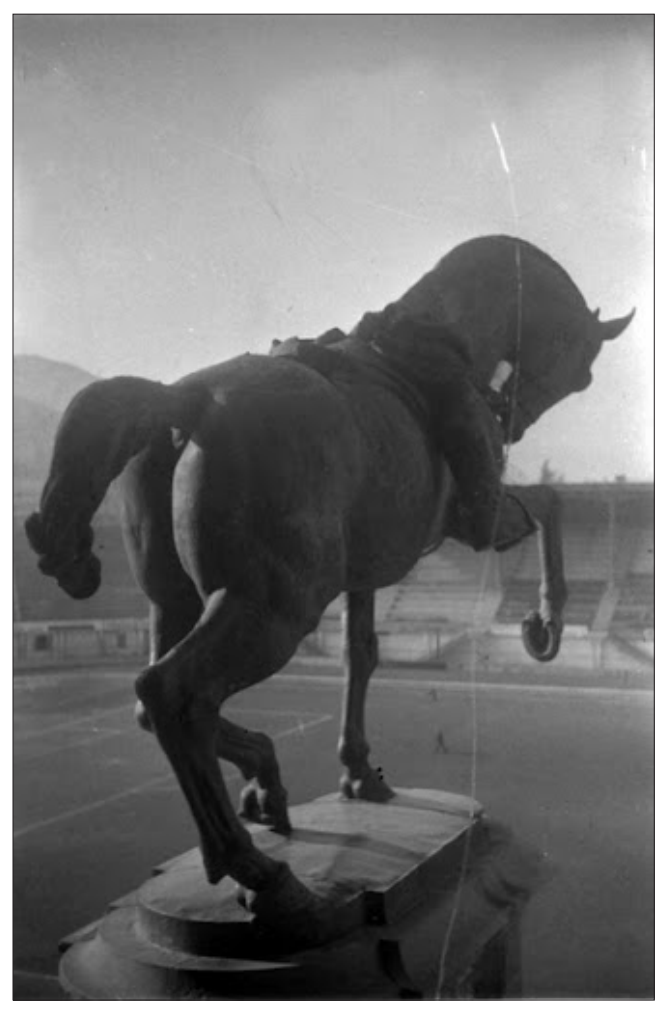

i simboli del fascismo divengono "incandescenti” in alcuni luoghi già dopo il 25 luglio 1943, in altri alla Liberazione.

Il caso, già citato, della statua di Mussolini a cavallo a Bologna è particolarmente interessante non solo per la sua precocità, ma perché rende ragione del potere dei simboli, fra trasformazione e risemantizzazione. La statua venne realizzata dallo scultore Giuseppe Graziosi nel 1929 fondendo due cannoni austriaci conquistati l'8 agosto 1848 dai bolognesi insorti. Collocata sotto la torre di Maratona nello stadio Littoriale, la figura di Mussolini venne disarcionata a furor di popolo il 26 luglio 1943, lasciando attaccato al basamento solo il cavallo con gli stivali del duce sui fianchi. La testa fu allora trascinata attraverso la città fino a scomparire, avvolta da leggende (si dice salvata da fascisti e preservata, ora, da nostalgici), il busto pare venne rifuso dal tedeschi dopo l'8 settembre, mentre il cavallo e gli stivali solo dopo il 1945 vennero a loro volta fusi per diventare il partigiano e la partigiana dello scultore Luciano Minguzzi, attualmente collocati a Porta Lame, per ricordare la battaglia del 7 novembre 1944 [Storchi 2013] ${ }^{16}$. Lo stesso materiale

16 Si veda anche Quattro matti dietro a una palla. Il primo secolo del Bologna Football Club nelle raccolte documentarie dell'Archiginnasio (http://badigit.comune.bologna.it/mostre/bologna fc/bacheca10.htm), mostra a cura di Maurizio Avanzolini e Marcello Fini, 20 maggio-26 settembre 2009. 


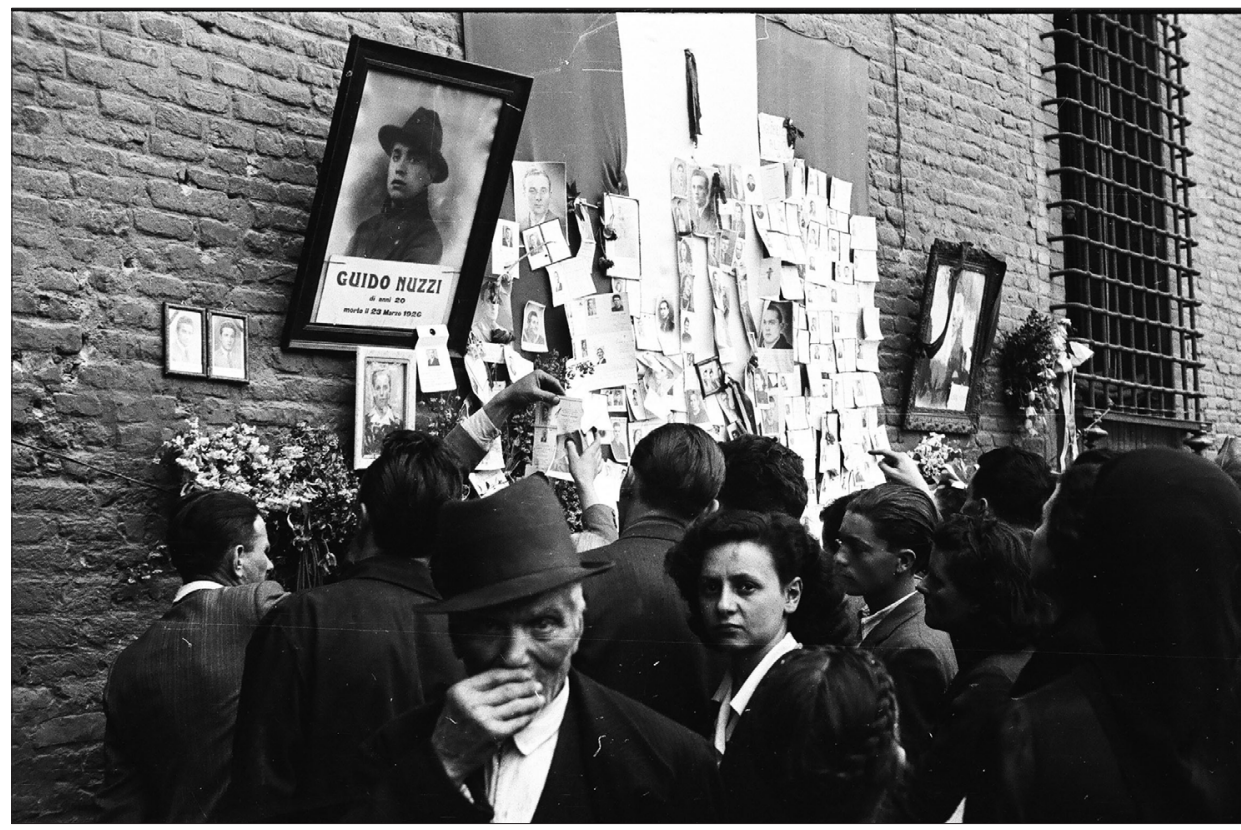

Fig. 14. La costruzione spontanea del sacrario dei caduti partigiani a Bologna, 21 aprile 1945 [foto Ed Reep].

è forgiato, distrutto e riplasmato, ogni volta dando forma al potere del presente. Ogni passaggio rappresenta una trasformazione politica e un livello storico ${ }^{17}$.

Non è, invece, diventato "incandescente" il monumento per l' "eroe coloniale" di Parma, l'esploratore Vittorio Bottego, da più di un secolo collocato davanti alla stazione ferroviaria. Realizzato nel 1907 per volontà di un comitato cittadino, ha attraversato l'Italia liberale, il fascismo, la Repubblica, avvolto fino a tempi recentissimi da una percezione tesa più all'epica che alla revisione critica del passato. Tuttavia, l'iconografia non lascia dubbi: la statua di Bottego domina le personificazioni dei due fiumi, l'Omo e il Giuba, che attraversano il territorio da lui esplorato, raffigurati come due guerrieri africani sconfitti. Di nuovo, un corpo bianco si impone su due corpi neri sottomessi, senza destare particolari rimostranze fino all'estate 2020, quando il monumento a Bottego ha iniziato a essere incluso nelle mappature della decolonizzazione culturale del paese [Scego 2020; Wu Ming 2 2021].

I monumenti, la statue, le lapidi che costituiscono la retorica pubblica attualmente visibile nel territorio di questa regione sono quelle nate dalla Resistenza e dalla Liberazione, non senza dissidi, dibattiti e discussioni. È una produzione di monumenti e statue sofferta, incentrata su figure di martiri più che di eroi, con cippi

17 Sullo stesso solco si colloca la trasformazione del monumento al legionario di Imola in uno al partigiano. In questo caso è lo stesso artista, Angelo Biancini, a portare i necessari cambiamenti: il gladio viene sostituito dal fucile, mentre sulle rocce del basamento restano le incongrue stilizzazioni di antilopi, che destano domande tuttavia in poche persone. 
e lapidi che segnano il territorio in modo capillare, testimoniando la pervasività dell'occupazione tedesca e dell'operato della Repubblica sociale italiana (Rsi). Sono i segni in cui la popolazione, in prevalenza, si riconosce e si unisce ${ }^{18}$. In molti casi, sono nati da urgenze dal basso, come il sacrario dei caduti in piazza del Nettuno a Bologna: il 21 aprile 1945, alla liberazione della città, i bolognesi riempirono il muro del palazzo del Comune, usato come luogo di fucilazioni dalla Rsi, con le foto dei loro cari. La folla di persone, in questo caso, non abbatte un monumento, ma ne crea uno: un monumento effimero e per questo straordinariamente incisivo, come descrive Jean Giono nella citazione in esergo. Quello che lo scrittore francese coglie con estrema chiarezza è che i monumenti, per essere sentiti, non devono essere fatti in materiali eterni, ma devono essere vivi per le persone che li vedono, abitano, attraversano ogni giorno. La loro possibilità di esistenza deriva dal fatto di corrispondere alle persone che li guardano.

Allontanandosi sempre più dalla fine del secondo conflitto mondiale e dalla Liberazione, questo tema ha progressivamente perso centralità nella produzione di nuovi monumenti anche in una regione, come l'Emilia-Romagna, che ha focalizzato la sua attenzione e narrazione pubblica sulla commemorazione della Resistenza. A partire dagli anni Novanta in questo territorio si delinea, dopo la crisi ideologica e culturale determinata dal 1989, una peculiarità degna di rilievo: iniziano a essere dedicate statue e monumenti a sportivi. Ayrton Senna, Marco Pantani, Dorando Pietri: figure in cui si uniscono gli elementi epici dello sport, ma anche quelli sacrificali. Nuovi eroi - e martiri - e nuovi casi di memorie tragiche, discusse e controverse, come indica soprattutto il caso di Pantani.

Infine, è necessario aggiungere una nota: in questa lunga ricognizione emerge tuttavia un' assenza. Abbiamo sempre parlato di uomini di marmo e bronzo, mai di donne. Le donne reali, che non siano figure simboliche, allegoriche, esornative o, peggio, ammiccanti forme sensuali e sessualizzate, sono praticamente assenti. In parallelo alle proteste per abbattere ciò che non calza più, i simboli in cui non ci si vuole più identificare, i modelli non più ammissibili per l'immedesimazione, altre voci e iniziative si sono levate per sottolineare questa mancanza. Anche in questo caso si tratta di operazioni dal basso, per lo più di attiviste-artiste, che stanno cercando di colmare questa imperdonabile assenza, creando un nuovo immaginario urbano, aggiornato a nuovi linguaggi formali, che travalicano le statue e i monumenti. Un immaginario che sia femminile, e femminista.

\section{Bibliografia}

Belpoliti 2011

Marco Belpoliti, Le teste rotolano, in «Doppiozero», 24 agosto 2011, https://www. doppiozero.com/materiali/editoriale/le-teste-rotolano.

18 Anche se, in particolare negli ultimi anni, sono proprio questi monumenti, in Emilia-Romagna, a subire attacchi di matrice politica. 


\section{Ben-Ghiat 2017}

Ruth Ben-Ghiat, Why Are so Many Fascist Monuments still Standing in Italy?, in «The New Yorker», 5 ottobre 2017, https://www.newyorker.com/culture/culture-desk/why-are-so-many-fascist-monuments-still-standing-in-italy.

Bianchi, Scego 2014

Rino Bianchi, Igiaba Scego, Roma negata. Percorsi postcoloniali nella città, Roma, Ediesse, 2014.

Burch-Brown 2020

Joanna Burch-Brown, Should Slavery's Statues Be Preserved? On Transitional Justice and Contested Heritage, in «Journal of Applied Philosophy», 20 novembre 2020, https://doi.org/10.1111/japp.12485.

Ciatti 2020

Marco Ciatti, Della distruzione di monumenti di personaggi storici, in «Opificio delle pietre dure», 13 luglio 2020, http://www.opificiodellepietredure.it/index.php?it/21/ news/728/della-distruzione-di-monumenti-di-personaggi-storici.

De Martino 1949

Ernesto De Martino, Intorno a una storia del mondo popolare e subalterno, in «Società», 5 (1949), pp. 411-435.

Di Michele 2020

Andrea Di Michele, Storicizzare i monumenti fascisti. Il caso di Bolzano, in "Geschichte und Region/Storia e regione», 29, 2 (2020), pp. 149-167.

Freedberg 1989

David Freedberg, The Power of Images. Studies in the History and Theory of Response, Chicago, The University of Chicago Press, 1989.

Franzen, König, Plath 2007

Sculpture Projects Muenster 07, a cura di Brigitte Franzen, Kasper König, Carina Plath, Köln, Walther König, 2007.

Franzoni 2020

Claudio Franzoni, Distruggere le statue: una storia antica, in «Doppiozero», 25 giugno 2020, https://www.doppiozero.com/materiali/distruggere-le-statue-una-storiaantica.

Gamboni 1997

Dario Gamboni, The Destruction of Art. Iconoclasm and Vandalism since the French Revolution, London, Reaktion Books, 1997.

Gamboni 2002

Dario Gamboni, Image to Destroy, Indestructible Image, in Latour, Weibel 2002, pp. 88-135.

Giono 1954

Jean Giono, Voyage en Italie, Paris, Gallimard, 1954.

Grechi, Lombardo 2021

Giulia Grechi, Salvo Lombardo, Anche le statue muoiono, dossier «Roots\&Routes. Research on Visual Culture», 35 (2021).

Herz 1992

Rudolf Herz, Lenins Lager. Entwurf für eine Skulptur in Dresden. Dokumentation, Berlin, Karin Kramer, 1992.

Latour, Weibel 2002

Iconoclash. Beyond the Image-Wars in Science, Religion and Art, a cura di Bruno Latour, Peter Weibel, Karlsruhe-Cambridge, ZKM and MIT Press, 2002. 
Layard 2020

Antonia Layard, Edward Colston: Listing Controversy, in «University of Bristol Law School Blog», 15 giugno 2020, https://legalresearch.blogs.bris.ac.uk/2020/06/ edward-colston-listing-controversy/.

Layard 2021

Antonia Layard, Listing Controversy II: Statues, Contested Heritage and the Policy of 'Retain and Explain', in «University of Bristol Law School Blog», 25 gennaio 2021, https://legalresearch.blogs.bris.ac.uk/2021/01/listing-controversy-ii-statuescontested-heritage-and-the-policy-of-retain-and-explain/.

Leech 2018

Patrick Leech, The Anxieties of Dissonant Heritage: ATRIUM and the Architectural Legacy of Regimes in Local and European Perspectives, in Architecture as Propaganda in Twentieth-Century Totalitarian Regimes: History and Heritage, a cura di Håkan Hökerberg, Firenze, Polistampa, 2018, pp. 245-260.

Lorini 2018

Alessandra Lorini, Il ritorno della Confederazione sudista: la guerra dei monumenti, il mito della supremazia bianca e l'uso pubblico della memoria della Guerra Civile americana, intervento alla seconda conferenza dell'Associazione italiana di public history, Pisa, 11-15 giugno 2018.

Lorini 2020

Alessandra Lorini, Il mito fondativo di Colombo, in «Jacobin Italia», 12 ottobre 2020, https://jacobinitalia.it/il-mito-fondativo-di-colombo.

Maida 2020

Desirée Maida, Statua di Indro Montanelli imbrattata a Milano. Tutte le volte che la scultura ha creato dissensi, in «Artribune», 14 giugno 2020, https://www.artribune. com/arti-visive/arte-contemporanea/2020/06/statua-di-indro-montanelli-imbrattataa-milano-tutte-le-volte-che-la-scultura-ha-creato-dissensi.

Macdonald 2009

Sharon Macdonald, Difficult Heritage: Negotiating the Nazi Past in Nuremberg and beyond, London, Routledge, 2009.

Mauri 1991

Paolo Mauri, Uomini e statue, in «La Repubblica», 30 agosto 1991, https://ricerca. repubblica.it/repubblica/archivio/repubblica/1991/08/30/uomini-statue.html.

McKinney 2018

Debra McKinney, Stone Mountain: A Monumental Dilemma, in «The Southern Poverty Law Center», 10 febbraio 2018, https://www.splcenter.org/fighting-hate/intelligence-report/2018/stone-mountain-monumental-dilemma.

Mirzoeff 2004

Nicholas Mirzoeff, Guardare la guerra. Immagini del potere globale, Roma, Meltemi 2004 (ed. or. 2004).

Mitterhofer 2013

Johanna Mitterhofer, Competing Narratives on the Future of Contested Heritage: A Case Study of Facists Monuments in Contemporary South Tyrol, Italy, «Heritage \& Society», 1 (2013), pp. 46-61.

Musil 2004

Robert Musil, Monumenti, in Pagine postume pubblicate in vita, Torino, Einaudi (ed. or. 1936; I ed. it. 1970), pp. 62-66. 


\section{Oliver 2018}

Samantha Oliver, No Offense to Robert Musil, but... The Continuing Relevance of Monuments, in «Public Seminar», 6 aprile 2018, https://publicseminar.org/2018/04/ no-offense-to-robert-musil-but.

Olusoga 2020

David Olusoga, The Toppling of Edward Colston's Statue Is not an Attack on History. It Is History, in «The Guardian», 8 giugno 2020, www.theguardian.com/commen www.theguardian.com/commentisfree/2020/jun/08/edward-colston-statue-historyslave-trader-bristol-protest tisfree/2020/jun/08/edward-colston-statue-history-slavetrader-bristol-protest.

Panico 2017

Mario Panico, Esplosioni di icone. Street art e iconoclastia performativa sui monumenti socialisti dell'Europa orientale, in «Ocula», 18 (2017): Street art: iconoclastia $e$ istituzionalizzazione, https://www.ocula.it/metadata.php?id=450.

Parkinson 2015

Justin Parkinson, Why Is Cecil Rhodes Such a Controversial Figure?, in «BBC News», 1 aprile 2015, https://www.bbc.com/news/magazine-32131829.

Pintarelli 2018

Flavio Pintarelli, Quello che resta dei monumenti fascisti, in «Il Tascabile», 13 giugno 2018, https://www.iltascabile.com/societa/monumenti-fascisti.

Pirazzoli 2010

Elena Pirazzoli, A partire da ciò che resta. Forme memoriali dal 1945 alle macerie del Muro di Berlino, Reggio Emilia, Diabasis, 2010.

Piretto 2014

Memorie di pietra. I monumenti delle dittature, a cura di Gian Piero Piretto, Milano, Raffaello Cortina, 2014.

Portelli 2020

Alessandro Portelli, Il ginocchio sul collo. L'America, il razzismo, la violenza tra presente, storia e immaginari, Roma, Donzelli, 2020.

Riegl 1985

Alois Riegl, Il culto moderno dei monumenti. Il suo carattere e i suoi inizi, a cura di Sandro Scarrocchia, Renate Trost, Bologna, Nuova Alfa, 1985 (ed. or. 1903).

Scego 2020

Igiaba Scego, Cosa fare con le tracce scomode del nostro passato, in «Internazionale», 9 giugno 2020, https://www.internazionale.it/opinione/igiaba-scego/2020/06/09/ tracce-passato-colonialismo-razzismo-fascismo.

Scotini 2014

Il piedistallo vuoto. Fantasmi dall'Est Europa, Catalogo della mostra tenuta a Bologna, Museo civico archeologico, 24 gennaio-16 marzo 2014, a cura di Marco Scotini, Milano, Mousse Publishing, 2014.

Staiti 2018

Claudio Staiti, Il «Columbus Circle» e i conti con il passato. Storia di un monumento che divide New York, intervento alla seconda conferenza dell'Associazione italiana di public history, Pisa, 11-15 giugno 2018.

Storchi 2013

Simona Storchi, Mussolini as Monument: The Equestrian Statue of the Duce at the Littoriale Stadium in Bologna, in The Cult of the Duce. Mussolini and the Italians, a cura di Stephen Gundle, Christopher Duggan, Giuliana Pieri, Manchester, MUP, 2013, pp. 193-208. 
Thompson 2020

Erin L. Thompson, Why just 'Adding Context' to Controversial Monuments May not Change Minds, in «Smithsonian Magazine», 18 dicembre 2020, https://www.smithsonianmag.com/history/why-just-adding-context-controversial-monument-may-notchange-minds-180976583.

Thompson 2021

Erin L. Thompson, The South's Monuments Will Rise again, in «The Washington Post», 5 marzo 2021, https://www.washingtonpost.com/opinions/2021/03/05/confederate-monuments-removed-temporarily.

Volquardsen 2020

Ebbe Volquardsen, Kulturhistoriker: Sådan bør vi afgøre, om statuer er bevaringsvcerdige, in "Altinget», 7 luglio 2020, https://www.altinget.dk/artikel/lektor-statuer-skalbidrage-til-den-positive-fortaelling-om-et-faelles-vi.

Wu Ming 22020

Wu Ming 2, Il vittimismo fascista nelle città italiane: storia di una lapide, 18 novembre 2020, https://www.wumingfoundation.com/giap/2020/11/il-vittimismo-fascistanelle-citta-italiane-storia-di-una-lapide/\#more-45803.

Wu Ming 22021

Wu Ming 2, Una mappa per ricordare i crimini del colonialismo italiano, in «Internazionale», 15 febbraio 2021, https://www.internazionale.it/opinione/wu-ming-2/ 2021/02/15/mappa-colonialismo-italiano.

Young 1992

James E. Young, The Counter-Monument: Memory against Itself in Germany Today, in «Critical Inquiry», 2 (1992), pp. 267-296.

Young 2000

James E. Young, At Memory's Edge. After-Images of the Holocaust in Contemporary Art and Architecture, New Haven-London, Yale University Press 2000.

Younge 2021

Gary Younge, Why Every Single Statue Should Come down. Statues of Historical Figures Are Lazy, Ugly and Distort History. From Cecil Rhodes to Rosa Parks, Let's Get Rid of Them All, 1 giugno 2021, https://www.theguardian.com/artanddesign/2021/ jun/01/gary-younge-why-every-single-statue-should-come-down-rhodes-colston.

Zucchetti 2020

Emilio Zucchetti, Di statue, performance moltitudinaria e memoria collettiva, in «Menelique», (2020), https://www.menelique.com/statue-blm.

\section{Risorse}

Progetto Atrium

https://www.atriumroute.eu

Collettivo Resistenze in Cirenaica https://resistenzeincirenaica.com

Progetto Temporary Museum of Modern Marx https://www.ufg.at/temporary-museum-of-modern-marx.4186.0.html

Database del «Washington Post» su uccisioni da parte delle forze di polizia negli Usa https://www.washingtonpost.com/graphics/investigations/police-shootings-database/

Mostra Enthüllt. Berlin und seine Denkmäler (Svelata. Berlino e i suoi monumenti) https://www.zitadelle-berlin.de/museen/enthullt/. 